\title{
Comparative analysis of the PET and ICT sensor properties of 1,8-naphthalimides containing aza-15-crown-5 ether moiety
}

\author{
Pavel A. Panchenko ${ }^{a, *}$, Yuri V. Fedorov ${ }^{a}$, Olga A. Fedorova ${ }^{a}$, Gediminas Jonusauskas ${ }^{b}$ \\ a A. N. Nesmeyanov Institute of Organoelement Compounds of Russian Academy of Sciences (INEOS RAS), 119991, Vavilova str. 28, Moscow, Russia \\ ${ }^{\mathrm{b}}$ Laboratoire Ondes et Matière d'Aquitaine (LOMA), UMR CNRS 5798, Bordeaux 1 University, 33405, Cours de la Libération 351, Talence, France
}

\section{A R T I C L E I I N F O}

\section{Keywords:}

1,8-naphthalimide

Crown compounds

Sensors

Photoinduced electron transfer (PET)

Internal charge transfer (ICT)

Optical spectroscopy

\begin{abstract}
A B S T R A C T
Novel 4 amino and 4 (acetyl)amino $N$ aryl 1,8 naphthalimides containing aza 15 crown 5 ether receptor unit in the $N$ aryl fragment and at $C 4$ of the naphthalimide residue were designed and prepared. Significant internal charge transfer from electron donating amino or amido group at $C 4$ of the naphthalene ring to the acceptor carboxyimide moiety as well as photoinduced electron transfer between $N$ aryl receptor and the naphthalimide fragment was revealed by the UV/Vis absorption spectroscopy and considerable fluorescence quenching. The addition of calcium perchlorate to an acetonitrile solution of naphthalimides with the receptor at imide nitrogen hindered the photoinduced electron transfer process and accordingly restored the quenched fluorescence of the free ligands. In the case of the compound in which the aza 15 crown 5 receptor is located at $\mathrm{C} 4$, the coordination with $\mathrm{Ca}^{2+}$ reduced the internal charge transfer interaction in the chromophore and caused a significant blue shift of the absorption and emission peak. The observed spectral effects were analyzed using PM6 semiempirical calculations. Formation of complexes was also confirmed by ${ }^{1}$ H NMR spectroscopy.
\end{abstract}

\section{Introduction}

Detecting cations is of great interest to many scientists, including chemists, biologists, clinical biochemists and environmentalists. With this regard, the development of fluorescent and colorimetric chemosensors seems to be advantageous, since the optical spec troscopy has turned to be one the most effective and reliable analytical methods [1]. Optical sensor devices are generally multi component systems comprising a signaling moiety (chromophore) and cation binding site (usually termed as receptor). The compo nents are chosen so that the complexation event triggers the modulation of photophysical characteristics of the chromophore such as the position of emission/absorption band maxima or the fluorescence intensity. Noteworthy, the mechanism of binding signal transduction between the sensor components may differ depending on the sensor design and may involve the photoinduced electron transfer (PET), the internal charge transfer (ICT), the reso nance energy transfer or the formation of excimer/exciplex [2-5].

1,8 Naphthalimides bearing electron donating substituents in the naphthalene moiety are well known organic fluorophores showing good photostability, large Stokes shifts and strong

\footnotetext{
* Corresponding author. Tel.: +7 49913580 98; fax: +7 4991355085 .

E-mail address: pavel@ineos.ac.ru (P.A. Panchenko).
}

absorption and emission in the UV/Vis region. Such compounds have been used in a large variety of areas, including coloration of polymers and optical brightening [6-8], laser active media [9,10], luminescent crack detection [11], electroluminescent materials [12-14], anticancer agents [15], liquid crystal displays [16,17] and fluorescent markers in biology [18-20]. The ability of 1,8 naphthalimide derivatives to be very sensitive to their surround ing render them as solvatochromic probes where the effects of solvent polarity on the electronic emission and absorption spectra are studied [21,22].

Owing to the unique feature of the naphthalimide chromophore that consists in its electron accepting properties, and thus possibility of photoinduced electron/energy transfer process or excimer/exci plex formation in the excited state, a significant number of naph thalimides have been developed as fluorescent chemosensors for metal cations and protons. $\mathrm{pH}$ Dependent fluorescence was found for piperazine substituted 4 aminonaphthalimides, which were config ured as « fluorophore-spacer-receptor » systems playing on the PET ON-OFF response [23,24]. Wang et al. synthesized a fluorescent naphthalimide based PET sensor for $\mathrm{Zn}^{2+}$ with di 2 picolylamine as a recognizing group [25]. Typically to ICT sensors, 4 piperidine naph thalimide derivatives containing 2 imino oxazolidin (thiazolidin) side chain at the imide moiety exhibited strong fluorescence quenching and some red shift in weakly acidic conditions, caused by the formation of an intramolecular $\mathrm{H}$ bond [26]. A selective and 
ratiometric $\mathrm{Cu}^{2+}$ fluorescent probe based on naphthalimide exci mer-monomer switching was designed by Xu et al. [27].

Even though crown ether receptors have been extensively used in the construction of fluorescent probes, there have been only few examples of naphthalimide chemosensors bearing crown ether groups as a receptor unit [28-33]. Recently, we studied the cation dependent fluorescent properties of benzo 15 crown 5 containg 4 amino and 4 (acetyl)amino $N$ aryl 1,8 naphthalimides $1 \mathrm{c}$ and 2c (Scheme 1) [34]. It was shown that the complex formation with the alkaline earth metal cations $\left(\mathrm{Mg}^{2+}, \mathrm{Ba}^{2+}\right)$ results in enhance ment of emission intensity in the case of 4 (acetyl)amino naphthalimide 2c due to inhibition of the PET process, which is originally operated in the free ligand. In contrast, 2a demonstrated the lack of fluorescence PET switching upon the coordination with metal ions.

Herein, we report on the synthesis and investigation of complexation properties of aza 15 crown 5 substituted naph thalimides $\mathbf{1 b}, \mathbf{2 b}$ and 3a. Considering the general principles used in the design of fluorescent probes [2-4], there exist two opportu nities for combination of azacrown ether receptor and naph thalimide chromophore. In the compounds $\mathbf{1 b}$ and $\mathbf{2 b}$, the receptor is attached to the $N$ aryl moiety. Alternatively, the aza 15 crown 5 may be presented in the molecule as a substituent at $C 4$ of the naphthalimide residue in a manner ensuring the conjugation be tween the receptor's amino nitrogen and carbonyl groups of the carboxyimide functionality, the feature implemented by us in the design of sensor $\mathbf{3 a}$. In such a case cation detection is possible not only by monitoring of emission intensity but also position of ab sorption and fluorescence maxima, which may be of interest for the development of ratiometric probes.

\section{Experimental}

\subsection{Materials and general methods}

4 Nitro 1,8 naphthalic anhydride $[35,36] 4,4$ bromo $N$ phenyl 1,8 naphthalimide 9 [37] and $N$ (4 aminophenyl)aza 15 crown 5 ether 7 [38] were prepared by literature procedures. All other re agents were purchased from commercial sources and were of the highest grade. Solvents were purified and dried according to stan dard procedures. Calcium perchlorate was dried in vacuum (7-8 $\mathrm{mm}$ $\mathrm{Hg}$ ) at $240{ }^{\circ} \mathrm{C}$ and kept anhydrous over $\mathrm{P}_{2} \mathrm{O}_{5}$ in desiccator (Caution! Calcium perchlorate may explode when heating. It decomposes at $270{ }^{\circ} \mathrm{C}$ [39]). Raney nickel catalyst was prepared from nickel aluminum alloy (weight percentage of nickel is 50\%) according to the known procedure [40].

Melting points were measured on Melt temp melting point electrothermal apparatus and were uncorrected. The reaction course and purity of the final products was followed by TLC on silica gel (DC Alufolien Kieselgel $60 \mathrm{~F}_{254}$, Merck). Column chromatog raphy was conducted over silica gel (Kieselgel 60, particle size $0.063-0.200 \mathrm{~mm}$, Merck) using as an eluent the gradient solvent mixtures benzene - ethanol and hexane - ethylacetate. FT IR spectra were recorded on Magna IR 750 Nicolet spectrometer and were obtained with potassium bromide pellets. Elemental analyses were carried out in the Microanalysis Laboratory of the A.N. Nes meyanov Institute of Organoelement Compounds.

\subsubsection{NMR measurements}

${ }^{1} \mathrm{H}$ and ${ }^{13} \mathrm{C}$ NMR spectra were recorded on an Avance 300, Avance 400 and Avance 600 spectrometers (Bruker) operating at $300.13,400.13,600.22 \mathrm{MHz}\left(\right.$ for ${ }^{1} \mathrm{H}$ ) and 75.47, 100.61, $150.93 \mathrm{MHz}$ (for ${ }^{13} \mathrm{C}$ ) respectively. The measurements were performed in DMSO $d_{6}, \mathrm{CDCl}_{3}$ and $\mathrm{CD}_{3} \mathrm{CN}$ solutions. The chemical shifts (given as $\delta$ ) were determined with an accuracy of $0.01 \mathrm{ppm}$ relative to the signals corresponding to the residual solvents and recalculated to the internal standard (TMS); the spin-spin coupling constants $(J)$ were measured with an accuracy of $0.1 \mathrm{~Hz}$. The numbering of carbon atoms in the naphthalimide moiety, $N$ aryl ring and crown ether fragment of compounds $\mathbf{1 b}, \mathbf{2 b}, \mathbf{3 a}, \mathbf{5}$ and $\mathbf{6}$ commonly used by us for the description of ${ }^{1} \mathrm{H}$ and ${ }^{13} \mathrm{C}$ NMR spectra is shown on Figs. 2, 5 and 8 . The assignment of ${ }^{1} \mathrm{H}$ and ${ }^{13} \mathrm{C}$ signals is based on $2 \mathrm{D}$ NMR experiments (HMBC, HSQC, ${ }^{1} \mathrm{H}$ COSY), which were performed using standard pulse sequences from the Bruker library. 2D NMR spectra are presented in Supplementary data.

\subsubsection{Mass spectrometry}

LC ESI MS analyses were performed on a Finnigan LCQ Advan tage mass spectrometer equipped with octopole ion trap mass analyzer, MS Surveyor pump, Surveyor auto sampler, Schmidlin Lab nitrogen generator (Germany) and Finnigan X Calibur 1.3 software for data collecting and processing. Acetonitrile (Panreac, HPLC gradient grade) was used as the mobile phase. Dilute MeCN solutions of compounds $\mathbf{1 b}, \mathbf{2} \mathbf{b}, \mathbf{3 a}$ and $\mathbf{5}$ were studied in the presence of 1 eq. of $\mathrm{HClO}_{4}$. The isocratic elution was maintained at a flow rate of $50 \mu \mathrm{L} \cdot \mathrm{min}^{-1}$ without column. The effluent from LC was passed directly into the electrospray ion source without a split. Positive electrospray ionization was achieved using a ionization voltage at $3 \mathrm{kV}$ with temperature at $200{ }^{\circ} \mathrm{C}$. Isotope patterns were calculated with Molecular Weight Calculator, Version 6.37 (Matthew Monroe).

Electron impact (EI) (70 eV) mass spectra were obtained from Finnigan Polaris Q instrument (ion trap) in standard conditions.

\subsubsection{Optical measurements}

The absorption spectra were taken on a Varian Cary 5G spec trophotometer. The fluorescence quantum yield measurements were performed using a Varian Cary 5G spectrophotometer and a FluoroMax 3 spectrofluorimeter. Spectral measurements were carried out in air saturated acetonitrile solutions (acetonitrile of spectrophotometric grade, water content $<0.005 \%$, Aldrich) at $20 \pm 1{ }^{\circ} \mathrm{C}$; the concentrations of studied compounds were of about $0.5-2.0 \cdot 10^{-5} \mathrm{M}$. All measured fluorescence spectra were cor rected for the nonuniformity of detector spectral sensitivity.<smiles>[R]c1ccc2c3c(cccc13)C(=O)N([R])C2=O</smiles>
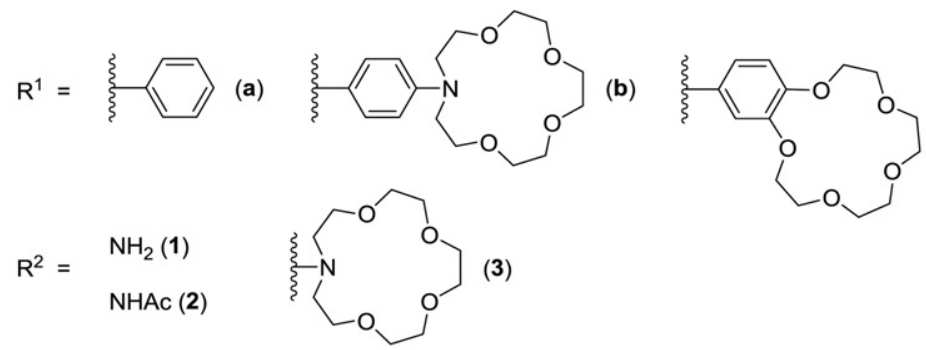

(c)

Scheme 1. Chemical structure of the compounds 1a c, 2a c and 3a. 
Coumarin 481 in acetonitrile ( $\varphi^{\mathrm{fl}} \quad 0.08$ ) [41] was used as reference for the fluorescence quantum yield measurements. The fluores cence quantum yields were calculated by the equation (1) [42]:

$\varphi_{\mathrm{i}}^{\mathrm{fl}} \quad \varphi_{0}^{\mathrm{fl}} \frac{S_{\mathrm{i}}\left(1-10^{-D_{0}}\right) n_{\mathrm{i}}^{2}}{S_{0}\left(1-10^{-D_{\mathrm{i}}}\right) n_{0}^{2}}$

wherein $\varphi_{\mathrm{i}}^{\mathrm{fl}}$ and $\varphi_{0}^{\mathrm{fl}}$ are the fluorescence quantum yields of the studied solution and the standard compound, respectively; $D_{\mathrm{i}}$ and $D_{0}$ are the absorptions of the studied solution and the standard, respectively; $S_{\mathrm{i}}$ and $S_{0}$ are the areas underneath the curves of the fluorescence spectra of the studied solution and the standard, respectively; and $n_{\mathrm{i}}$ and $n_{0}$ are the refraction indices of the solvents for the substance under study and the standard compound $\left(n_{\mathrm{i}} \quad n_{0} \quad 1.342\right.$, acetonitrile).

\subsubsection{Equilibrium constant determination}

Complex formation of dyes $\mathbf{1 b}, \mathbf{2} \mathbf{b}$ and $\mathbf{3 a}$ with $\mathrm{Ca}^{2+}$ in aceto nitrile at $20 \pm 1{ }^{\circ} \mathrm{C}$ was studied by spectrofluorometric (for $\mathbf{1 b}, \mathbf{2 b}$ and 3a) and spectrophotometric (for 3a) titration [43,44]. The ratio of dye to $\mathrm{Ca}^{2+}$ was varied by adding aliquots of a solution of $\mathrm{Ca}\left(\mathrm{ClO}_{4}\right)_{2}$ of known concentration to a solution of ligands $\mathbf{1 b}, \mathbf{2} \mathbf{b}$ or 3a of known concentration. The fluorescence (absorption) spec trum of each solution was recorded, and the stability constants of the complexes were determined using the SPECFIT/32 program (Spectrum Software Associates, West Marlborough, MA). The following equilibria were considered in the fitting (equations (1) and (2), L 1b, 2b or 3a; $\mathrm{M} \quad \mathrm{Ca}^{2+}$ ): In doing so, it was found that the experimental data corresponded to the theoretical ones if only the equation (2) was taken into account and the formation of the complexes with composition of 2:1 was not observed.

$\mathrm{L}+\mathrm{M} \rightleftharpoons \mathrm{LM}$

$2 \mathrm{~L}+\mathrm{M} \rightleftharpoons \mathrm{L}_{2} M$

The equilibrium constants for protonation of ligands $\mathbf{1 b}$ and $\mathbf{2 b}$ were not determined by this method because of high stability $\left(K>10^{7} \mathrm{M}^{1}\right)$ of protonated forms $(\mathbf{1 b}) \cdot \mathrm{H}^{+}$and $(\mathbf{2 b}) \cdot \mathrm{H}^{+}$.

\subsubsection{Determination of fluorescence quantum yields of complexes}

The fluorescence quantum yields of complexes $(\mathbf{1 b}) \cdot \mathrm{Ca}^{2+}$ and $(\mathbf{2 b}) \cdot \mathrm{Ca}^{2+}$ were determined using solutions of ligands $\mathbf{1 - 2 b}$ in $\mathrm{CH}_{3} \mathrm{CN}$ containing an excess of $\mathrm{Ca}\left(\mathrm{ClO}_{4}\right)_{2}$ in order to obtain $90-95 \%$ of ligand bound with the cation. The required $\mathrm{Ca}\left(\mathrm{ClO}_{4}\right)_{2}$ excess was calculated from the known stability constants using the SPECFIT/32 program. The measurements of $\varphi^{\mathrm{fl}}$ for $(\mathbf{1 b}) \cdot \mathrm{H}^{+}$and $(\mathbf{2 b}) \cdot \mathrm{H}^{+}$were done in the presence of 2 eq. $\mathrm{HClO}_{4}$ in ligand solutions, which can be understood from the fact that the further addition of $\mathrm{HClO}_{4}$ did not result in fluorescence enhancement and complex formation had already been finished. In the case of complex $(\mathbf{3 a}) \cdot \mathrm{Ca}^{2+}$, the direct quantum yield determination was impossible because the presence of considerable amount of the free ligand 3a which appreciably contributes the measured total absorption at $\lambda_{\text {ex }}$ and integrated emission signal might not be avoided even at high excess of calcium cation. The quantum yield of $(\mathbf{3 a}) \cdot \mathrm{Ca}^{2+}$ complex was estimated using the known quantum yield of $\mathbf{3 a}$ and the ratio of areas underneath the calculated fluorescence spectra of the in dividual components $\mathbf{3 a}$ and $(\mathbf{3 a}) \cdot \mathrm{Ca}^{2+}$.

\subsection{6. $X$ ray diffraction analysis}

Orange crystals of compound $\mathbf{1 b}$ were prepared by slow evapo ration of solution of $\mathbf{1 b}$ in the mixture of benzene and ethanol $(v /$

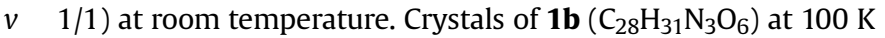
are monoclinic, space group Cc, $a$ 13.801(2), b 14.290(2), c $\quad 12.990(2) \AA, \beta \quad 98.634(4)^{\circ}, V \quad 2532.9(5) \AA^{3}, Z \quad 4, M \quad 505.56$, $d_{\text {calc }} \quad 1.326 \mathrm{~g} \mathrm{~cm}^{-3}, \mu(\mathrm{MoK} \alpha) \quad 0.94 \mathrm{Cm}^{-1}, F(000) \quad 1072$. Intensities of 7584 reflections were measured with a Smart APEX2 CCD diffractometer at $100 \mathrm{~K}\left(\lambda(\mathrm{MoK} \alpha) \quad 0.71072 \AA\right.$, $\omega$ scans with $0.3^{\circ}$ step, $\left.2 \theta<58^{\circ}\right)$, and 3356 independent reflections ( $\left.R_{\text {int }} \quad 0.0425\right)$ were used in further refinement. The structure was solved by direct method and refined by the full matrix least squares technique against $F^{2}$ in the anisotropic isotropic approximation. Hydrogen atoms of $\mathrm{NH}_{2}$ group were located from the Fourier synthesis while all others were placed in calculated positions and refined in the riding model. The refinement converged to $w R_{2} \quad 0.0950$ and GOF $\quad 1.009$ for all independent reflections $\left(\begin{array}{ll}R_{1} & 0.0460 \text { was }\end{array}\right.$ calculated against $F$ for 2595 observed reflections with $I>2 \sigma(I))$. All calculations were performed using SHELX software package [45]. Crystallographic data for the structure of $\mathbf{1 b}$ has been deposited with the Cambridge Crystallographic Data Centre as supplemental pub lication CCDC 916216. These data can be obtained free of charge from The Cambridge Crystallographic Data Centre through www.ccdc. cam.ac.uk/data_request/cif.

\subsubsection{Computational details}

Quantum chemical calculations were carried out by the MOPAC 2009 program package using the PM6 semiempirical method [46]. $\mathrm{CI}$ calculations were performed at optimized geometries, which reached gradient variations less than $0.01 \mathrm{kcal} / \mathrm{mol}$. The solvent effect was included in geometry optimizations following the "COnductorlike Screening MOdel" (COSMO) implemented in MOPAC 2009. A dielectric constant of $\varepsilon \quad 40$ and a refraction index of solvent $(n)$ such that $n^{2} \quad 2$ were used. The CI included eight occupied and eight unoccupied MOs.

\subsection{Synthesis of the compounds}

\subsubsection{2 (4 (1,4,7,10 Tetraoxa 13 azacyclopentadecan 13 yl) phenyl) 6 amino $1 \mathrm{H}$ benzo[d,e]isoquinoline 1,3(2H) dione $(\mathbf{1 b})$}

Method A. To a solution of compound $5(242 \mathrm{mg}, 0.452 \mathrm{mmol})$ in 1,4 dioxane $(10.3 \mathrm{~mL})$ hydrazine hydrate $(1.8 \mathrm{~mL}, 100 \mathrm{wt}$ \%) was added at $90{ }^{\circ} \mathrm{C}$. Raney nickel catalyst prepared from $1.5 \mathrm{~g}$ of nickel aluminum alloy was added portionwise to the reaction mass over a period of $30 \mathrm{~min}$. The resulting mixture was stirred for $1.5 \mathrm{~h}$ at $90^{\circ} \mathrm{C}$ (TCL control in a solvent system benzene - ethanol, $v / v$ 5/1) and then was filtered hot. The filtrate was evaporated under vacuum. The crude product was purified by column chromatography on silica gel using the gradient solvent mixture benzene - ethanol and then recrystallized from ethanol. Yield $112 \mathrm{mg}$ (49\%). M. p. 282$284{ }^{\circ} \mathrm{C} .{ }^{1} \mathrm{H}$ NMR $\left(600.22 \mathrm{MHz}\right.$, DMSO $\left.d_{6}, 31^{\circ} \mathrm{C}\right): \delta \quad 3.51-3.60(\mathrm{~m}$, $16 \mathrm{H} ; \mathrm{CH}_{2}(15), \mathrm{CH}_{2}(17), \mathrm{CH}_{2}(18), \mathrm{CH}_{2}(19), \mathrm{CH}_{2}(20), \mathrm{CH}_{2}(21), \mathrm{CH}_{2}(22)$, $\left.\mathrm{CH}_{2}(24)\right), \quad 3.67-3.72\left(\mathrm{~m}, 4 \mathrm{H} ; \quad \mathrm{CH}_{2}(16), \mathrm{CH}_{2}(23)\right), 6.70$ (d, $\left.{ }^{3} J(\mathrm{H}, \mathrm{H}) \quad 8.9,2 \mathrm{H} ; \mathrm{H}(11), \mathrm{H}(13)\right), 6.87\left(\mathrm{~d},{ }^{3} \mathrm{~J}(\mathrm{H}, \mathrm{H}) \quad 8.5,1 \mathrm{H} ; \mathrm{H}(3)\right)$, 7.01 (d, $\left.{ }^{3} \mathrm{~J} \quad 8.9,2 \mathrm{H} ; \mathrm{H}(10), \mathrm{H}(14)\right), 7.40$ (br s, $2 \mathrm{H} ; \mathrm{NH}_{2}$ ), 7.67 (dd, $\left.{ }^{3} J(\mathrm{H}, \mathrm{H}) \quad 7.3,{ }^{3} \mathrm{~J}(\mathrm{H}, \mathrm{H}) \quad 8.6,1 \mathrm{H} ; \mathrm{H}(6)\right), 8.18\left(\mathrm{~d},{ }^{3} \mathrm{~J}(\mathrm{H}, \mathrm{H}) \quad 8.5,1 \mathrm{H}\right.$, $\mathrm{H}(2)), 8.41\left(\mathrm{~d},{ }^{3} \mathrm{~J}(\mathrm{H}, \mathrm{H}) \quad 7.3,1 \mathrm{H} ; \mathrm{H}(7)\right), 8.64\left(\mathrm{~d},{ }^{3} \mathrm{~J}(\mathrm{H}, \mathrm{H}) \quad 8.6,1 \mathrm{H}\right.$; $\mathrm{H}(5)) .{ }^{13} \mathrm{C}$ NMR $\left(100.61 \mathrm{MHz}\right.$, DMSO $\left.d_{6}, 27{ }^{\circ} \mathrm{C}\right): \delta 52.10\left(\mathrm{CH}_{2}(15)\right.$, $\left.\mathrm{CH}_{2}(24)\right), 67.93\left(\mathrm{CH}_{2}(16), \mathrm{CH}_{2}(23)\right), 69.12\left(\mathrm{CH}_{2}(19), \mathrm{CH}_{2}(20)\right), 69.45$ $\left(\mathrm{CH}_{2}(17), \mathrm{CH}_{2}(22)\right), 70.38\left(\mathrm{CH}_{2}(21), \mathrm{CH}_{2}(18)\right), 108.05$ (C(1)), 108.16 (C(3)), 110.86 (C(11), $C(13)), 119.49$ (C(4a)), 122.40 (C(8)), 124.00 (C(6)), 124.18 (C(9)), $129.31(C(5)), 129.56$ (C(10), $C(14)), 130.08$ (C(8a)), $131.07(C(7)), 133.95(C(2)), 146.70(C(12)), 152.65(C(4))$, 163.51 (C(8b)), 164.35 (C(8c)). FT IR (KBr), $\mathrm{cm}^{-1}: 3355,3415,3255$ $\left(v_{\mathrm{NH} 2}\right)$; 3080, $2865\left(v_{\mathrm{CH}}\right), 1680,1635\left(v_{\mathrm{N}} \mathrm{C}=0\right)$. ESI MS in MeCN, calculated for $[\mathrm{M}+\mathrm{H}]^{+}, m / z$ : 506.23; found: 506.19. Elemental analysis: calculated (\%) for $\mathrm{C}_{28} \mathrm{H}_{31} \mathrm{~N}_{3} \mathrm{O}_{6}$ (MW 505.56): $\mathrm{C} 66.53, \mathrm{H}$ $6.14, \mathrm{~N} 8.32$; found $\mathrm{C} 66.56, \mathrm{H} 6.10, \mathrm{~N}$ 8.27. Method $B$. To a suspension of 4 nitronaphthalimide $\mathbf{5}$ (68 $\mathrm{mg}, 0.127 \mathrm{mmol})$ in ethanol $(1.0 \mathrm{~mL})$ a solution of $\mathrm{SnCl}_{2} \cdot 2 \mathrm{H}_{2} \mathrm{O}(100 \mathrm{mg})$ in concd hydrochloric acid 
( $0.5 \mathrm{~mL}, \rho \quad 1.18 \mathrm{~g} / \mathrm{mL}$ ) was added dropwise and the mixture was stirred at ambient temperature for $2 \mathrm{~h}$. The reaction mixture was poured into $\mathrm{NaOH}$ water solution $(7.0 \mathrm{~mL}, 5$ wt. \%). The brown precipitate was filtered off, washed with distilled water, dried at $60{ }^{\circ} \mathrm{C}$ and then chromatographed on silica gel using gradient ben zene - ethanol mixture as an eluent. Consequently, two fractions were isolated: fraction 1 (10 mg) and fraction 2 (14 mg). Fraction 1 was found to be 3 chloro 4 aminonaphthalimide $\mathbf{6}$ (the data for the compound $\mathbf{6}$ are listed below), fraction 2 - target product $\mathbf{1 b}$ (yield 22\%). M. p. $264-272{ }^{\circ} \mathrm{C} .{ }^{1} \mathrm{H}$ NMR (300.13 MHz, DMSO $\left.d_{6}, 23{ }^{\circ} \mathrm{C}\right)$ : $\delta \quad 3.46-3.63\left(\mathrm{~m}, 16 \mathrm{H} ; \mathrm{CH}_{2}(15), \mathrm{CH}_{2}(17), \mathrm{CH}_{2}(18), \mathrm{CH}_{2}(19)\right.$, $\left.\mathrm{CH}_{2}(20), \mathrm{CH}_{2}(21), \mathrm{CH}_{2}(22), \mathrm{CH}_{2}(24)\right), 3.64-3.75$ (m, 4H; $\mathrm{CH}_{2}(16)$, $\left.\mathrm{CH}_{2}(23)\right), 6.69\left(\mathrm{~d},{ }^{3} \mathrm{~J}(\mathrm{H}, \mathrm{H}) \quad 8.9,2 \mathrm{H} ; \mathrm{H}(11), \mathrm{H}(13)\right), 6.86$ (d, $\left.{ }^{3} J(\mathrm{H}, \mathrm{H}) \quad 8.5,1 \mathrm{H} ; \mathrm{H}(3)\right), 7.01\left(\mathrm{~d},{ }^{3} J(\mathrm{H}, \mathrm{H}) \quad 8.9,2 \mathrm{H} ; \mathrm{H}(10), \mathrm{H}(14)\right)$, 7.44 (br s, 2H; $\mathrm{NH}_{2}$ ), 7.67 (dd, $1 \mathrm{H},{ }^{3} J(\mathrm{H}, \mathrm{H}) \quad 7.3,{ }^{3} \mathrm{~J}(\mathrm{H}, \mathrm{H}) \quad 8.6 ; \mathrm{H}(6)$ ), $8.18\left(\mathrm{~d},{ }^{3} J(\mathrm{H}, \mathrm{H}) \quad 8.5,1 \mathrm{H} ; \mathrm{H}(2)\right), 8.41\left(\mathrm{dd},{ }^{4} J(\mathrm{H}, \mathrm{H}) \quad 0.7,{ }^{3} J(\mathrm{H}, \mathrm{H}) \quad 7.3\right.$, $1 \mathrm{H} ; \mathrm{H}(7)), 8.64\left(\mathrm{dd},{ }^{4} J(\mathrm{H}, \mathrm{H}) \quad 0.7,{ }^{3} J(\mathrm{H}, \mathrm{H}) \quad 8.6,1 \mathrm{H} ; \mathrm{H}(5)\right)$. EI MS, $m /$ $z$ (I, \%): 506 (19), 505 (59) [M] ${ }^{+}, 449$ (29), 386 (34), 360 (46), 330 (54), 316 (100), 303 (34), 196 (36), 195 (81).

\subsection{2. $N$ (2 (4 (1,4,7,10 Tetraoxa 13 azacyclopentadecan 13 yl) phenyl) 1,3 dioxo 2,3 dihydro $1 \mathrm{H}$ benzo[d,e]isoquinolin $6 \mathrm{yl}$ ) acetamide (2b)}

To a suspension of compound $\mathbf{1 b}(40 \mathrm{mg}, 0.079 \mathrm{mmol})$ in an anhydrous dichloromethane $(1.0 \mathrm{~mL})$ acetylchloride $(60 \mu \mathrm{L}$, $0.844 \mathrm{mmol}$ ) was added. The reaction mass was refluxed and stir red for $3 \mathrm{~h}$ to afford a clear solution. After the completion of the reaction, the mixture was diluted with ethanol $(1.0 \mathrm{~mL})$. The sol vents were removed under vacuum and $\mathrm{Na}_{2} \mathrm{CO}_{3}$ water solution (5.0 mL, 10 wt. \%) was added to the residual solid to neutralize $\mathrm{HCl}$. Product 2b was extracted with dichloromethane, the extract was washed with distilled water and evaporated under vacuum. The resulting solid $(38 \mathrm{mg}$ ) was recrystallized from ethanol to afford $33 \mathrm{mg}$ of pure 2b (yield 76\%). M. p. $114-117{ }^{\circ} \mathrm{C} .{ }^{1} \mathrm{H}$ NMR (300.13 MHz, DMSO $\left.d_{6}, 21^{\circ} \mathrm{C}\right): \delta \quad 2.30\left(\mathrm{~s}, 3 \mathrm{H} ; \mathrm{CH}_{3}\right), 3.48-3.64(\mathrm{~m}$, $16 \mathrm{H} ; \mathrm{CH}_{2}(15), \mathrm{CH}_{2}$ (17), $\mathrm{CH}_{2}(18), \mathrm{CH}_{2}(19), \mathrm{CH}_{2}(20), \mathrm{CH}_{2}(21), \mathrm{CH}_{2}(22)$, $\left.\mathrm{CH}_{2}(24)\right)$, 3.64-3.75 (m, 4H; $\left.\mathrm{CH}_{2}(16), \mathrm{CH}_{2}(23)\right), 6.71$ (d, $2 \mathrm{H}$, $\left.{ }^{3} J(\mathrm{H}, \mathrm{H}) \quad 8.9 ; \mathrm{H}(11), \mathrm{H}(13)\right), 7.09\left(\mathrm{~d},{ }^{3} J(\mathrm{H}, \mathrm{H}) \quad 8.9,2 \mathrm{H} ; \mathrm{H}(10), \mathrm{H}(14)\right)$, 7.85-7.97 (m, 1H; H(6)), $8.33\left(\mathrm{~d}, 1 \mathrm{H},{ }^{3} \mathrm{~J}(\mathrm{H}, \mathrm{H}) \quad 8.2 ; \mathrm{H}(3)\right), 8.47(\mathrm{~d}, 1 \mathrm{H}$, $\left.{ }^{3} J(\mathrm{H}, \mathrm{H}) \quad 8.2 ; \mathrm{H}(2)\right), 8.52\left(\mathrm{~d},{ }^{3} \mathrm{~J}(\mathrm{H}, \mathrm{H}) \quad 7.3,1 \mathrm{H} ; \mathrm{H}(7)\right), 8.74(\mathrm{~d}$, $\left.{ }^{3} J(\mathrm{H}, \mathrm{H}) \quad 8.6,1 \mathrm{H} ; \mathrm{H}(5)\right), 10.44$ (br s, $\left.1 \mathrm{H} ; \mathrm{NHCO}\right) .{ }^{13} \mathrm{C}$ NMR (150.93 MHz, DMSO $\left.d_{6}, 21{ }^{\circ} \mathrm{C}\right): \delta \quad 24.09\left(\mathrm{CH}_{3}\right), 52.12\left(\mathrm{CH}_{2}(15)\right.$, $\left.\mathrm{CH}_{2}(24)\right), 67.91\left(\mathrm{CH}_{2}(16), \mathrm{CH}_{2}(23)\right), 69.12\left(\mathrm{CH}_{2}(19), \mathrm{CH}_{2}(20)\right), 69.47$ $\left(\mathrm{CH}_{2}(17), \mathrm{CH}_{2}(22)\right), 70.38\left(\mathrm{CH}_{2}(18), \mathrm{CH}_{2}(21)\right), 110.93$ (C(11), $\left.\mathrm{C}(13)\right)$, 118.12 (C(1)), $119.44(\mathrm{C}(3)), 122.96(\mathrm{C}(8)), 123.54(\mathrm{C}(9)), 124.16$ $(C(4 a)), 126.40(C(6)), 128.71(C(8 a)), 129.23(C(5)), 129.47(C(10)$, $\mathrm{C}(14)), 130.88(\mathrm{C}(7)), 131.61(\mathrm{C}(2)), 140.26(\mathrm{C}(4)), 146.96(\mathrm{C}(12))$, $163.51(\mathrm{C}(8 \mathrm{~b})), 164.07(\mathrm{C}(8 \mathrm{c})), 169.62\left(\mathrm{CH}_{3} \mathrm{CO}\right) . \mathrm{FT}$ IR $(\mathrm{KBr}), \mathrm{cm}^{-1}$ : $3350\left(v_{\mathrm{NH}}\right) ; 2920,2855\left(v_{\mathrm{CH}}\right) ; 1705,1660\left(v_{\mathrm{N}} \mathrm{C}=0\right) ; 1520\left(\delta_{\mathrm{NH}}\right)$. ESI MS in MeCN, calculated for $[\mathrm{M}+\mathrm{H}]^{+}, m / z$ : 548.24; found: 548.18 . Elemental analysis: calculated (\%) for $\mathrm{C}_{30} \mathrm{H}_{33} \mathrm{~N}_{3} \mathrm{O}_{7}$ (MW 547.60): $\mathrm{C}$ 65.80, H 6.07, N 7.67; found C 65.71, H 6.06, N 7.62.

\subsubsection{Phenyl $6(1,4,7,10$ tetraoxa 13 azacyclopentadecan 13}

yl) $1 \mathrm{H}$ benzo $[$ d,e] isoquinoline $1,3(2 \mathrm{H})$ dione $(\mathbf{3 a})$

A mixture of 4 bromo $N$ phenyl 1.8 naphthalimide 9 (422 mg, $1.199 \mathrm{mmol}$ ) and aza 15 crown 5 ether (988 $\mathrm{mg}, 4.511 \mathrm{mmol}$ ) was heated to reflux in methoxylethanol $(3 \mathrm{~mL})$ under argon atmo sphere for $8 \mathrm{~h}$ (TCL control in a solvent system benzene - ethanol, $v / v \quad 5 / 1)$. After removal of methoxylethanol, the solid was purified by silica gel column chromatography using benzene - ethanol gradient mixture as an eluent. The weight of isolated product $\mathbf{3 a}$ was $220 \mathrm{mg}$ (yield 37\%). M. p. $149-150{ }^{\circ} \mathrm{C} .{ }^{1} \mathrm{H}$ NMR $(300.13 \mathrm{MHz}$, DMSO $\left.d_{6}, 21^{\circ} \mathrm{C}\right), \delta \quad 3.52-3.67\left(\mathrm{~m}, 16 \mathrm{H} ; \mathrm{CH}_{2}(15), \mathrm{CH}_{2}(17), \mathrm{CH}_{2}(18)\right.$, $\left.\mathrm{CH}_{2}(19), \mathrm{CH}_{2}(20), \mathrm{CH}_{2}(21), \mathrm{CH}_{2}(22), \mathrm{CH}_{2}(24)\right)$, 3.68-3.77 (m, 4H;
$\mathrm{CH}_{2}$ (16), $\left.\mathrm{CH}_{2}(23)\right), 7.30-7.38(\mathrm{~m}, 2 \mathrm{H} ; \mathrm{H}(10), \mathrm{H}(14)), 7.41-7.58$ (m, 4H; H(3), H(11), H(12), H(13)), 7.79 (dd, ${ }^{3} J(\mathrm{H}, \mathrm{H}) \quad 7.3,{ }^{3} J(\mathrm{H}, \mathrm{H}) \quad 8.5$, $1 \mathrm{H} ; \mathrm{H}(6)), 8.36\left(\mathrm{~d},{ }^{3} J(\mathrm{H}, \mathrm{H}) \quad 8.2,1 \mathrm{H} ; \mathrm{H}(2)\right), 8.46\left(\mathrm{dd},{ }^{4} J(\mathrm{H}, \mathrm{H}) \quad 0.7\right.$, $\left.{ }^{3} \mathrm{~J}(\mathrm{H}, \mathrm{H}) \quad 7.3,1 \mathrm{H} ; \mathrm{H}(7)\right), 8.81\left(\mathrm{dd},{ }^{4} \mathrm{~J}(\mathrm{H}, \mathrm{H}) \quad 0.7,{ }^{3} \mathrm{~J}(\mathrm{H}, \mathrm{H}) \quad 8.5,1 \mathrm{H}\right.$; $\mathrm{H}(5)) .{ }^{13} \mathrm{C}$ NMR $\left(100.61 \mathrm{MHz}\right.$, DMSO $\left.d_{6}, 27{ }^{\circ} \mathrm{C}\right): \delta \quad 52.76\left(\mathrm{CH}_{2}(15)\right.$ $\left.\mathrm{CH}_{2}(24)\right), 68.66\left(\mathrm{CH}_{2}(16), \mathrm{CH}_{2}(23)\right), 69.85\left(2 \times \mathrm{CH}_{2}\right), 69.97$ $\left(2 \times \mathrm{CH}_{2}\right), 70.42\left(2 \times \mathrm{CH}_{2}\right), 114.93(\mathrm{C}(1)), 116.59(\mathrm{C}(3)), 122.86(\mathrm{C}(8))$, 125.42 (C(6)), 126.22 (C(4a)), 128.04 (C(12)), 128.81 (C(11), $C(13))$, 129.18 (C(10), $C(14)), 130.09$ (C(8a)), $130.69(C(7)), 131.67(C(2)$ or $C(5)), 131.76(C(5)$ or $C(2)), 136.27(C(9)), 155.41(C(4)), 163.28$ $(\mathrm{C}(8 \mathrm{~b})), 163.91(\mathrm{C}(8 \mathrm{c}))$. ESI MS in MeCN, calculated for $[\mathrm{M}+\mathrm{H}]^{+}, \mathrm{m} /$ $z$ : 491.22; found: 491.29. EI MS, $m / z$ (I, \%): 490 (32) [M] $]^{+}, 357$ (34), 341 (37), 316 (49), 315 (91), 314 (100), 313 (66), 301 (59), 299 (52), 288 (47), 208 (34), 196 (34). Elemental analysis: calculated (\%) for $\mathrm{C}_{28} \mathrm{H}_{30} \mathrm{~N}_{2} \mathrm{O}_{6}$ (MW 490.21): C 68.56, H 6.16, N 5.71; found C 68.49, H 6.19, N 5.68 .

\subsubsection{2 (4 (1,4,7,10 Tetraoxa 13 azacyclopentadecan 13 yl)}

phenyl) 6 nitro $1 \mathrm{H}$ benzo[d,e] isoquinoline 1,3(2H) dione (5)

A solution of a mixture of 4 nitro 1,8 naphthalic anhydride 4 (304 $\mathrm{mg}, 1.251 \mathrm{mmol}$ ) and $N$ (4 aminophenyl)aza 15 crown 5 ether $7(554 \mathrm{mg})$ in $80 \%$ acetic acid $(1.3 \mathrm{~mL})$ was kept at reflux for $1.5 \mathrm{~h}$. The reaction mass was cooled down; the crystals precipitated upon cooling were filtered off and washed on a filter with distilled water. The product was dried at $80{ }^{\circ} \mathrm{C}$ and purified by column chromatography on silica gel using the mixture of benzene ethanol ( $v / v \quad 50 / 1)$ as an eluent. The weight of the obtained product 5 was $401 \mathrm{mg}$ (yield 60\%). M. p. 227-229 ${ }^{\circ} \mathrm{C} .{ }^{1} \mathrm{H}$ NMR spectra $\left(400.13 \mathrm{MHz}\right.$, DMSO $\left.d_{6}, 50{ }^{\circ} \mathrm{C}\right): \delta \quad 3.49-3.62(\mathrm{~m}, 16 \mathrm{H}$; $\mathrm{CH}_{2}(15), \mathrm{CH}_{2}(17), \mathrm{CH}_{2}(18), \mathrm{CH}_{2}(19), \mathrm{CH}_{2}(20), \mathrm{CH}_{2}(21), \mathrm{CH}_{2}(22)$, $\left.\mathrm{CH}_{2}(24)\right), 3.65-3.74\left(\mathrm{~m}, 4 \mathrm{H} ; \mathrm{CH}_{2}(16), \mathrm{CH}_{2}(23)\right), 6.73$ (d, $\left.{ }^{3} J(\mathrm{H}, \mathrm{H}) \quad 8.7,2 \mathrm{H} ; \mathrm{H}(11), \mathrm{H}(13)\right), 7.13\left(\mathrm{~d},{ }^{3} J(\mathrm{H}, \mathrm{H}) \quad 8.7,1 \mathrm{H} ; \mathrm{H}(10)\right.$, $\mathrm{H}(14)), 8.08-8.17(\mathrm{~m}, 1 \mathrm{H} ; \mathrm{H}(6)), 8.58\left(\mathrm{~d},{ }^{3} \mathrm{~J}(\mathrm{H}, \mathrm{H}) \quad 8.2,1 \mathrm{H} ; \mathrm{H}(3)\right.$ ), $8.61\left(\mathrm{~d},{ }^{3} \mathrm{~J}(\mathrm{H}, \mathrm{H}) \quad 8.2,1 \mathrm{H} ; \mathrm{H}(2)\right), 8.63\left(\mathrm{~d},{ }^{3} \mathrm{~J}(\mathrm{H}, \mathrm{H}) \quad 7.3,1 \mathrm{H} ; \mathrm{H}(7)\right), 8.76$ $\left(\mathrm{d},{ }^{3} \mathrm{~J}(\mathrm{H}, \mathrm{H}) \quad 8.6,1 \mathrm{H} ; \mathrm{H}(5)\right) .{ }^{13} \mathrm{C}$ NMR spectra (75.47 MHz, DMSO $d_{6}$, $\left.21^{\circ} \mathrm{C}\right): \delta \quad 52.12\left(\mathrm{CH}_{2}(15), \mathrm{CH}_{2}(24)\right), 67.88\left(\mathrm{CH}_{2}(16), \mathrm{CH}_{2}(23)\right), 69.10$ $\left(\mathrm{CH}_{2}(19), \mathrm{CH}_{2}(20)\right), 69.48\left(\mathrm{CH}_{2}(17), \mathrm{CH}_{2}(22)\right), 70.38\left(\mathrm{CH}_{2}(21)\right.$, $\left.\mathrm{CH}_{2}(18)\right), 110.94(\mathrm{C}(11), \mathrm{C}(13)), 122.85(\mathrm{C}(4 \mathrm{a})), 122.99(\mathrm{C}(8)), 123.50$ $(\mathrm{C}(9)), 124.28(\mathrm{C}(3)), 127.42(\mathrm{C}(1)), 128.32(\mathrm{C}(8 \mathrm{a})), 128.72(\mathrm{C}(5))$, $129.33(C(10), C(14)), 129.57(C(2)), 130.14(C(6)), 131.69(C(7))$, 147.15 (C(12)), $149.15(C(4)), 162.73(C(8 b)), 163.55$ (C(8c)). FT IR $(\mathrm{KBr}) / \mathrm{cm}^{-1}: 3085,2865\left(v_{\mathrm{CH}}\right) ; 1715,1680\left(v_{\mathrm{N}} \mathrm{C}=0\right) ; 1525,1350$ $\left(v_{\mathrm{NO} 2}\right)$. ESI MS in MeCN, calculated for $[\mathrm{M}+\mathrm{H}]^{+}, m / z$ : 536.20; found: 536.13. Elemental analysis: calculated (\%) for $\mathrm{C}_{28} \mathrm{H}_{29} \mathrm{~N}_{3} \mathrm{O}_{8}$ (MW 535.55): C 62.80, H 5.46, N 7.85; found C 63.01, H 5.49, N 7.81.

\subsubsection{2 (4 (1,4,7,10 Tetraoxa 13 azacyclopentadecan 13 yl) phenyl) 6 amino 5 chloro $1 \mathrm{H}$ benzo[d,e]isoquinoline 1,3(2H) dione (6)}

According to the Method $B$ for the reduction of nitro group in the compound 5 (see above), $10 \mathrm{mg}$ of 4 amino 3 chloronaphthalimide 6 (yield 15\%) was obtained from $68 \mathrm{mg}$ of $5,100 \mathrm{mg}$ of $\mathrm{SnCl}_{2} \cdot 2 \mathrm{H}_{2} \mathrm{O}$, $0.5 \mathrm{~mL}$ of concd $\mathrm{HCl}$ and $1.0 \mathrm{~mL}$ of ethanol. M. p. $288-291{ }^{\circ} \mathrm{C} .{ }^{1} \mathrm{H}$ NMR (300.13 MHz, DMSO $\left.d_{6}, 23{ }^{\circ} \mathrm{C}\right): \delta$ 3.49-3.63 (m, 16H; $\mathrm{CH}_{2}(15), \mathrm{CH}_{2}(17), \mathrm{CH}_{2}(18), \mathrm{CH}_{2}(19), \mathrm{CH}_{2}(20), \mathrm{CH}_{2}(21), \mathrm{CH}_{2}(22)$, $\left.\mathrm{CH}_{2}(24)\right)$, 3.64-3.75 ( $\left.\mathrm{m}, 4 \mathrm{H} ; \mathrm{CH}_{2}(16), \mathrm{CH}_{2}(23)\right), 6.69$ (d, ${ }^{3} J(\mathrm{H}, \mathrm{H}) \quad 8.9$, 2H; H(11), H(13)), 7.03 (d, $\left.{ }^{3} J(\mathrm{H}, \mathrm{H}) \quad 8.9,2 \mathrm{H} ; \mathrm{H}(10), \mathrm{H}(14)\right), 7.55$ (br s, $\left.2 \mathrm{H} ; \mathrm{NH}_{2}\right), 7.76\left(\mathrm{dd}, 1 \mathrm{H},{ }^{3} J(\mathrm{H}, \mathrm{H}) \quad 7.3,{ }^{3} \mathrm{~J}(\mathrm{H}, \mathrm{H}) \quad 8.6 ; \mathrm{H}(6)\right), 8.21(\mathrm{~s}, 1 \mathrm{H}$; $\mathrm{H}(2)), 8.44$ (dd, $\left.{ }^{4} J(\mathrm{H}, \mathrm{H}) \quad 0.7,{ }^{3} J(\mathrm{H}, \mathrm{H}) \quad 7.3,1 \mathrm{H} ; \mathrm{H}(7)\right), 8.78$ (dd, $\left.{ }^{4} J(\mathrm{H}, \mathrm{H}) \quad 0.7,{ }^{3} J(\mathrm{H}, \mathrm{H}) \quad 8.6,1 \mathrm{H} ; \mathrm{H}(5)\right) .{ }^{13} \mathrm{C} \mathrm{NMR}(150.93 \mathrm{MHz}$, DMSO $\left.d_{6}, 25^{\circ} \mathrm{C}\right): \delta \quad 52.10\left(\mathrm{CH}_{2}(15), \mathrm{CH}_{2}(24)\right), 67.91\left(\mathrm{CH}_{2}(16), \mathrm{CH}_{2}(23)\right)$, $69.12\left(\mathrm{CH}_{2}(19), \mathrm{CH}_{2}(20)\right), 69.47\left(\mathrm{CH}_{2}(17), \mathrm{CH}_{2}(22)\right), 70.38\left(\mathrm{CH}_{2}(18)\right.$, $\left.\mathrm{CH}_{2}(21)\right), 109.48(\mathrm{C}(1)), 110.89$ (C(11), C(13)), 111.71 (C(3)), 120.29 $(C(4 a)), 122.74(C(8)), 123.76(C(9)), 125.40(C(6)), 128.35(C(8 a))$, $129.37(C(5)), 129.48(C(10), C(14)), 131.04(C(7)), 132.65(C(2))$, 
$146.82(C(12)), 147.51(C(4)), 162.85(C(8 b)), 164.03(C(8 c))$. EI MS, $m /$ $z(I, \%): 541(20)[\mathrm{M}+2]^{+}, 539\left([\mathrm{M}]^{+}\right)(55), 486(26), 420$ (29), 394 (49), 380 (37), 364 (59), 350 (100), 337 (38), 231 (28), 229 (75). Elemental analysis: calculated (\%) for $\mathrm{C}_{28} \mathrm{H}_{30} \mathrm{ClN}_{3} \mathrm{O}_{6}$ (MW 540.01): C 62.28, H 5.60, N 7.78; found C 62.34, H 5.65, N 7.74.

\subsection{NMR data for the free dyes and their complexes with $\mathrm{Ca}^{2+}$ in $\mathrm{CD}_{3} \mathrm{CN}$}

\subsubsection{Compound $\mathbf{1 b}$}

$C_{\mathrm{L}} \quad 6.8 \cdot 10^{-3} \mathrm{M} .{ }^{1} \mathrm{H}$ NMR $\left(300.13 \mathrm{MHz}, \mathrm{CD}_{3} \mathrm{CN}, 21^{\circ} \mathrm{C}\right): \delta \quad 3.50-$ 6.64 (m, 16H; $\mathrm{CH}_{2}$ (15), $\mathrm{CH}_{2}(24), \mathrm{CH}_{2}(17), \mathrm{CH}_{2}(22), \mathrm{CH}_{2}(18), \mathrm{CH}_{2}(21)$, $\left.\mathrm{CH}_{2}(19), \mathrm{CH}_{2}(20)\right)$, 3.69-3.78 (m, 4H; $\left.\mathrm{CH}_{2}(16), \mathrm{CH}_{2}(23)\right), 5.90$ (br s, $\left.2 \mathrm{H} ; \mathrm{NH}_{2}\right), 6.77\left(\mathrm{~d},{ }^{3} J(\mathrm{H}, \mathrm{H}) \quad 8.9,2 \mathrm{H} ; \mathrm{H}(11), \mathrm{H}(13)\right), 6.93$ (d, $\left.{ }^{3} J(\mathrm{H}, \mathrm{H}) \quad 8.3,1 \mathrm{H} ; \mathrm{H}(3)\right), 7.04\left(\mathrm{~d},{ }^{3} \mathrm{~J}(\mathrm{H}, \mathrm{H}) \quad 8.9,2 \mathrm{H} ; \mathrm{H}(10), \mathrm{H}(14)\right), 7.69$ $\left(\mathrm{dd},{ }^{3} \mathrm{~J}(\mathrm{H}, \mathrm{H}) \quad 7.3,{ }^{3} \mathrm{~J}(\mathrm{H}, \mathrm{H}) \quad 8.3,1 \mathrm{H} ; \mathrm{H}(6)\right), 8.27\left(\mathrm{~d},{ }^{3} \mathrm{~J}(\mathrm{H}, \mathrm{H}) \quad 8.3,1 \mathrm{H}\right.$; $\mathrm{H}(2)), 8.35\left(\mathrm{~d},{ }^{3} J(\mathrm{H}, \mathrm{H}) \quad 8.3,1 \mathrm{H} ; \mathrm{H}(7)\right), 8.49\left(\mathrm{~d},{ }^{3} \mathrm{~J}(\mathrm{H}, \mathrm{H}) \quad 7.3,1 \mathrm{H} ; \mathrm{H}(5)\right)$.

\subsubsection{Compound $\mathbf{2 b}$}

$C_{\mathrm{L}} \quad 1.0 \cdot 10^{-2} \mathrm{M}^{1}{ }^{1} \mathrm{H}$ NMR $\left(300.13 \mathrm{MHz}, \mathrm{CD}_{3} \mathrm{CN}, 2{ }^{\circ} \mathrm{C}\right): \delta \quad 2.30$ (s, 3H; $\mathrm{CH}_{3} \mathrm{CO}$ ), 3.53-6.65 (m, $16 \mathrm{H} ; \mathrm{CH}_{2}(15), \mathrm{CH}_{2}(24), \mathrm{CH}_{2}(17)$, $\left.\mathrm{CH}_{2}(22), \mathrm{CH}_{2}(18), \mathrm{CH}_{2}(21), \mathrm{CH}_{2}(19), \mathrm{CH}_{2}(20)\right), 3.70-3.78$ (m, 4H; $\left.\mathrm{CH}_{2}(16), \mathrm{CH}_{2}(23)\right), 6.79\left(\mathrm{~d},{ }^{3} \mathrm{~J}(\mathrm{H}, \mathrm{H}) \quad 8.9,2 \mathrm{H}, \mathrm{H}(11), \mathrm{H}(13)\right), 7.08$ (d, $\left.{ }^{3} J(\mathrm{H}, \mathrm{H}) \quad 8.9, \quad 2 \mathrm{H} ; \quad \mathrm{H}(10), \quad \mathrm{H}(14)\right), 7.83\left(\mathrm{dd},{ }^{3} \mathrm{~J}(\mathrm{H}, \mathrm{H}) \quad 7.2\right.$, $\left.{ }^{3} \mathrm{~J}(\mathrm{H}, \mathrm{H}) \quad 8.5,1 \mathrm{H} ; \mathrm{H}(6)\right), 8.31\left(\mathrm{~d},{ }^{3} \mathrm{~J}(\mathrm{H}, \mathrm{H}) \quad 8.2,1 \mathrm{H} ; \mathrm{H}(3)\right), 8.49(\mathrm{~d}$, $\left.{ }^{3} J(\mathrm{H}, \mathrm{H}) \quad 8.2,1 \mathrm{H} ; \mathrm{H}(2)\right), 8.52\left(\mathrm{~d},{ }^{3} \mathrm{~J}(\mathrm{H}, \mathrm{H}) \quad 8.5,1 \mathrm{H} ; \mathrm{H}(7)\right), 8.55(\mathrm{~d}$, $\left.{ }^{3} J(\mathrm{H}, \mathrm{H}) \quad 7.2,1 \mathrm{H} ; \mathrm{H}(5)\right) ; 8.88$ (br s, $\left.1 \mathrm{H} ; \mathrm{NHCO}\right)$

\subsubsection{Compound $3 a$}

$C_{\mathrm{L}} \quad 1.0 \cdot 10^{-2} \mathrm{M}^{1}{ }^{1} \mathrm{H}$ NMR $\left(300.13 \mathrm{MHz}, \mathrm{CD}_{3} \mathrm{CN}, 21^{\circ} \mathrm{C}\right): \delta \quad 3.55-$ 3.70 ( $\mathrm{m}, 16 \mathrm{H} ; \mathrm{CH}_{2}$ (15), $\mathrm{CH}_{2}(24), \mathrm{CH}_{2}(17), \mathrm{CH}_{2}(22), \mathrm{CH}_{2}(18), \mathrm{CH}_{2}(21)$, $\left.\mathrm{CH}_{2}(19), \mathrm{CH}_{2}(20)\right), 3.71-3.80$ (m, 4H; $\left.\mathrm{CH}_{2}(16), \mathrm{CH}_{2}(23)\right), 7.27-7.38$ (m, 2H; H(10), H(14)), 7.42-7.59 (m, 4H; H(3), H(11), H(12), H(13)), $7.74\left(\mathrm{dd},{ }^{3} J(\mathrm{H}, \mathrm{H}) \quad 7.2,{ }^{3} J(\mathrm{H}, \mathrm{H}) \quad 8.6,1 \mathrm{H} ; \mathrm{H}(6)\right), 8.41\left(\mathrm{~d},{ }^{3} J(\mathrm{H}, \mathrm{H}) \quad 8.3\right.$, $1 \mathrm{H} ; \mathrm{H}(2)), 8.50\left(\mathrm{dd},{ }^{4} \mathrm{~J}(\mathrm{H}, \mathrm{H}) \quad 1.1,{ }^{3} \mathrm{~J}(\mathrm{H}, \mathrm{H}) \quad 7.2,1 \mathrm{H} ; \mathrm{H}(7)\right), 8.85(\mathrm{dd}$, $\left.{ }^{4} J(\mathrm{H}, \mathrm{H}) \quad 1.1,{ }^{3} J(\mathrm{H}, \mathrm{H}) \quad 8.6,1 \mathrm{H} ; \mathrm{H}(5)\right)$.

\subsubsection{Complex $(\mathbf{1 b}) \cdot \mathrm{Ca}^{2+}$}

$C_{\mathrm{L}} \quad 1.0 \cdot 10^{-2} \mathrm{M}$. Metal to ligand ratio $\mathrm{M}: \mathrm{L} \quad 3: 1 .{ }^{1} \mathrm{H}$ NMR (300.13 MHz, $\left.\mathrm{CD}_{3} \mathrm{CN}, 21{ }^{\circ} \mathrm{C}\right): \delta \quad 3.39-3.49\left(\mathrm{~m}, 4 \mathrm{H} ; \mathrm{CH}_{2}(15)\right.$, $\mathrm{CH}_{2}(24)$ ), 3.82-3.98 (m, 16H; $\mathrm{CH}_{2}(16), \mathrm{CH}_{2}(17), \mathrm{CH}_{2}(18), \mathrm{CH}_{2}(19)$, $\left.\mathrm{CH}_{2}(20), \mathrm{CH}_{2}(21), \mathrm{CH}_{2}(22), \mathrm{CH}_{2}(23)\right), 6.02$ (br s, $2 \mathrm{H} ; \mathrm{NH}_{2}$ ), 6.94 (d, $\left.{ }^{3} J(\mathrm{H}, \mathrm{H}) \quad 8.3,1 \mathrm{H} ; \mathrm{H}(3)\right), 7.34\left(\mathrm{~d},{ }^{3} \mathrm{~J}(\mathrm{H}, \mathrm{H}) \quad 8.9,2 \mathrm{H} ; \mathrm{H}(11), \mathrm{H}(13)\right)$, $7.45\left(\mathrm{~d},{ }^{3} \mathrm{~J}(\mathrm{H}, \mathrm{H}) \quad 8.9,2 \mathrm{H} ; \mathrm{H}(10), \mathrm{H}(14)\right), 7.70\left(\mathrm{dd},{ }^{3} \mathrm{~J}(\mathrm{H}, \mathrm{H}) \quad 7.3\right.$, $\left.{ }^{3} J(\mathrm{H}, \mathrm{H}) \quad 8.4,1 \mathrm{H} ; \mathrm{H}(6)\right), 8.28\left(\mathrm{~d},{ }^{3} \mathrm{~J}(\mathrm{H}, \mathrm{H}) \quad 8.3,1 \mathrm{H} ; \mathrm{H}(2)\right), 8.39$ (dd, $\left.{ }^{4} J(\mathrm{H}, \mathrm{H}) \quad 1.0,{ }^{3} J(\mathrm{H}, \mathrm{H}) \quad 8.4,1 \mathrm{H} ; \mathrm{H}(7)\right), 8.50\left(\mathrm{dd},{ }^{4} J(\mathrm{H}, \mathrm{H}) \quad 1.0\right.$ $3(\mathrm{H}, \mathrm{H}) \quad 7.3,1 \mathrm{H} ; \mathrm{H}(5))$.

\subsubsection{Complex $(\mathbf{2} \boldsymbol{b}) \cdot \mathrm{Ca}^{2+}$}

$C_{\mathrm{L}} \quad 1.0 \cdot 10^{-2} \mathrm{M}$. Metal to ligand ratio $\mathrm{M}: \mathrm{L} \quad 3: 1 .{ }^{1} \mathrm{H}$ NMR (300.13 MHz, $\left.\mathrm{CD}_{3} \mathrm{CN}, 21^{\circ} \mathrm{C}\right): \delta \quad 2.33\left(\mathrm{~s}, 3 \mathrm{H} ; \mathrm{CH}_{3} \mathrm{CO}\right), 3.41-3.50(\mathrm{~m}$, $4 \mathrm{H} ; \mathrm{CH}_{2}(15), \mathrm{CH}_{2}(24)$ ), 3.84-3.99 ( $\mathrm{m}, 16 \mathrm{H} ; \mathrm{CH}_{2}(16), \mathrm{CH}_{2}(17), \mathrm{CH}_{2}(18)$, $\left.\mathrm{CH}_{2}(19), \mathrm{CH}_{2}(20), \mathrm{CH}_{2}(21), \mathrm{CH}_{2}(22), \mathrm{CH}_{2}(23)\right), 7.36$ (d, ${ }^{3} \mathrm{~J}(\mathrm{H}, \mathrm{H}) \quad 8.9$, 2H; H(11), H(13)), 7.47 (d, $\left.{ }^{3} J(\mathrm{H}, \mathrm{H}) \quad 8.9,2 \mathrm{H} ; \mathrm{H}(10), \mathrm{H}(14)\right), 7.88$ (dd, $\left.{ }^{3} J(\mathrm{H}, \mathrm{H}) \quad 7.4,{ }^{3} J(\mathrm{H}, \mathrm{H}) \quad 8.4,1 \mathrm{H} ; \mathrm{H}(6)\right), 8.30\left(\mathrm{~d},{ }^{3} \mathrm{~J}(\mathrm{H}, \mathrm{H}) \quad 8.1,1 \mathrm{H} ; \mathrm{H}(3)\right)$, 8.48-8.64 (m, 3H; H(2), H(5), H(7)), 9.06 (br s, 1H; NHCO).

\subsubsection{Complex $(3 \boldsymbol{a}) \cdot \mathrm{Ca}^{2+}$}

$C_{\mathrm{L}} \quad 1.0 \cdot 10^{-2} \mathrm{M}$. Metal to ligand ratio M:L 5:1. ${ }^{1} \mathrm{H}$ NMR (300.13 MHz, $\left.\mathrm{CD}_{3} \mathrm{CN}, 21{ }^{\circ} \mathrm{C}\right): \delta \quad 3.64-3.73\left(\mathrm{~m}, 4 \mathrm{H} ; \mathrm{CH}_{2}(15)\right.$, $\mathrm{CH}_{2}(24)$ ), 3.76-3.91 (m, 16H; $\mathrm{CH}_{2}(16), \mathrm{CH}_{2}(17), \mathrm{CH}_{2}(18), \mathrm{CH}_{2}(19)$, $\left.\mathrm{CH}_{2}(20), \mathrm{CH}_{2}(21), \mathrm{CH}_{2}(22), \mathrm{CH}_{2}(23)\right), 7.30-7.39$ (m, 2H; $\mathrm{H}(10)$, $\mathrm{H}(14)), 7.45-7.60(\mathrm{~m}, 4 \mathrm{H} ; \mathrm{H}(3), \mathrm{H}(11), \mathrm{H}(12), \mathrm{H}(13)), 7.86$ (dd, $\left.{ }^{3} J(\mathrm{H}, \mathrm{H}) \quad 7.2,{ }^{3} \mathrm{~J}(\mathrm{H}, \mathrm{H}) \quad 8.6,1 \mathrm{H} ; \mathrm{H}(6)\right), 8.51\left(\mathrm{~d},{ }^{3} \mathrm{~J}(\mathrm{H}, \mathrm{H}) \quad 8.2,1 \mathrm{H}\right.$;
$\mathrm{H}(2)), 8.58\left(\mathrm{dd},{ }^{4} J(\mathrm{H}, \mathrm{H}) \quad 1.0,{ }^{3} J(\mathrm{H}, \mathrm{H})\right.$

7.2, $1 \mathrm{H} ; \mathrm{H}(7)), 8.78$ (dd,

\section{Results and discussion}

\subsection{Synthesis of crown containing naphthalimides}

Compounds $\mathbf{1 b}$ and $\mathbf{2 b}$ containing crown a ether fragment in the $N$ aryl moiety were prepared as shown in Scheme 2. Starting from 4 nitronaphthalic anhydride $\mathbf{4}$, the intermediate 4 nitro 1,8 napthalimide 5 was afforded by the reaction with $N$ (4 aminophenyl)aza 15 crown 5 ether 7 in AcOH media. The reduc tion of nitro group in $\mathbf{5}$ to yield $\mathbf{1 b}$ was performed by the hydrazine hydrate over Raney nickel catalyst in 1,4 dioxane solution. Unex pectedly, the use of tin (II) chloride with hydrochloric acid for the reduction didn't allow us to isolate compound 1b with high yield unlike the synthesis of 4 aminonaphthalides containing benzo 15 crown 5 ether groups we have described previously [47]. Moreover, we observed simultaneous introduction of chlorine atom in ortho position toward the amino group in this case. At the final step, the treatment of 1b with acetylchloride under anhydrous conditions resulted in 4 (acetyl)aminonaphthalimide $\mathbf{2 b}$.

Scheme 2 also shows synthesis of azacrown ether derivative 3a. According to the routine procedure [37], 4 bromonaphthalic an hydride 8 first was refluxed in glacial acetic acid with aniline. Subsequent nucleophilic substitution of the bromine atom in the isolated $N$ phenylimide $\mathbf{9}$ with aza 15 crown 5 ether yielded target aminonaphthalimide $\mathbf{3 a}$.

Amine $\mathbf{7}$ used in Scheme 2 is a known compound; it was ob tained from commercially available $N$ (phenyl)diethanolamine and triethylene glycol $\mathrm{di}(p$ toluenesulfonate) in a three step synthesis [38]. 4 Amino and 4 (acetyl)amino $N$ phenyl 1,8 naphthalimides 1a and 2a have been previously synthesized in our laboratory [47].

\subsection{Photophysical properties of the compounds}

Photophysical characteristics of the compounds $\mathbf{1 a}, \mathbf{1 b}, \mathbf{2 a}, \mathbf{2 b}$ and 3a were measured in acetonitrile solution and the data are presented in Table 1. As an example, Fig. 1 shows absorption and emission spectra of $N$ phenylnaphthalimides 1a, 2a and 3a. Simi larly to other 4 amino and 4 amidonaphthalimide derivatives $[48,49]$, the broad and structureless absorption band in the long wavelength region arises from the polarization of chromophore naphthalimide system due to the intramolecular charge transfer (ICT) interaction occurring between the electron donating

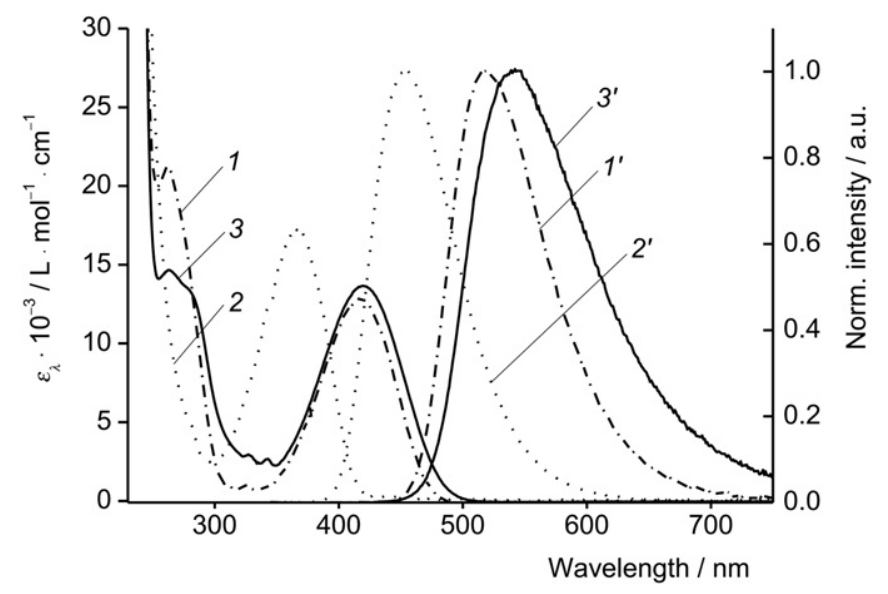

Fig. 1. Steady-state absorption $(1,2,3)$ and normalized emission $\left(1^{\prime}, 2^{\prime}, 3^{\prime}\right)$ spectra of the compounds $\mathbf{1 a}\left(1,1^{\prime}\right), \mathbf{2 a}\left(2,2^{\prime}\right)$ and $\mathbf{3 a}\left(3,3^{\prime}\right)$ in acetonitrile $\left(C \sim 10{ }^{5} \mathrm{M}\right)$. 


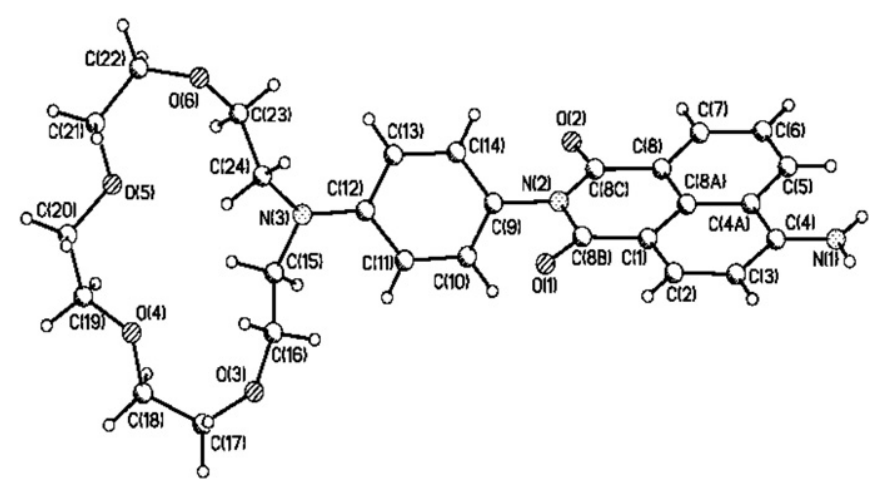

Fig. 2. X-ray crystallography data for the compound 1b. $C(8 b) \quad N(2) C(9) C(10)$ dihedral angle value is $84.3^{\circ}$.

substituents at $C(4)$ and the acceptor carboxyimide fragment. Substitution of amino hydrogen in $\mathbf{1 a}, \mathbf{1} \mathbf{b}$ by the acetyl group to produce 2a, 2b causes a blue shift of $\lambda_{\max }^{\mathrm{abs}}$ and $\lambda_{\max }^{\mathrm{fl}}$ with a concomitant changes in fluorescence quantum yields, which is a result of decreased donor ability of NHAc group relative to $\mathrm{NH}_{2}$.

The introduction of aza 15 crown 5 ether substituent at $C 4$ of the naphthalene ring when going from 1a to $\mathbf{3 a}$ is expected to enhance ICT interaction in the molecule because of inductive effect of two alkyl groups. Surprisingly, we observed only slight bath ochromic shift (3 nm) in the absorption spectra (Table 1, Fig. 1). As compared with compound 1a, the fluorescence maximum of $\mathbf{3 a}$ is $22 \mathrm{~nm}$ red shifted with the emission intensity dramatically decreased. This is very similar to the observations of Saha and Samanta [50] who studied the influence the structure of the amino group on the photophysical properties of 4 aminonaphthalimide derivatives. The small red shift in the absorption spectra can be understood if it is assumed that the dialkylamino group is twisted with respect to the naphthalimide plane in a manner ensuring the partial loss of steric repulsion between the azacrown ether sub stituent and the peri hydrogen atoms of naphthalene residue. Such repulsion disfavors charge separation and reduces the donor properties of azacrown ether substituent. In fact, the PM6 opti mized ground state geometry of the compound 3a depicted in Fig. S1 (see Supplementary data) confirms this assumption.

The relatively low fluorescence quantum yield of compound 3a with respect to $\mathbf{1 a}$ is also a result of conformational changes at the 4 amino moiety. As it has been proposed by Saha and Samanta [50], the increase in steric strain in the excited ICT state of 4 dialkylaminonaphthalimides, more planar in nature in

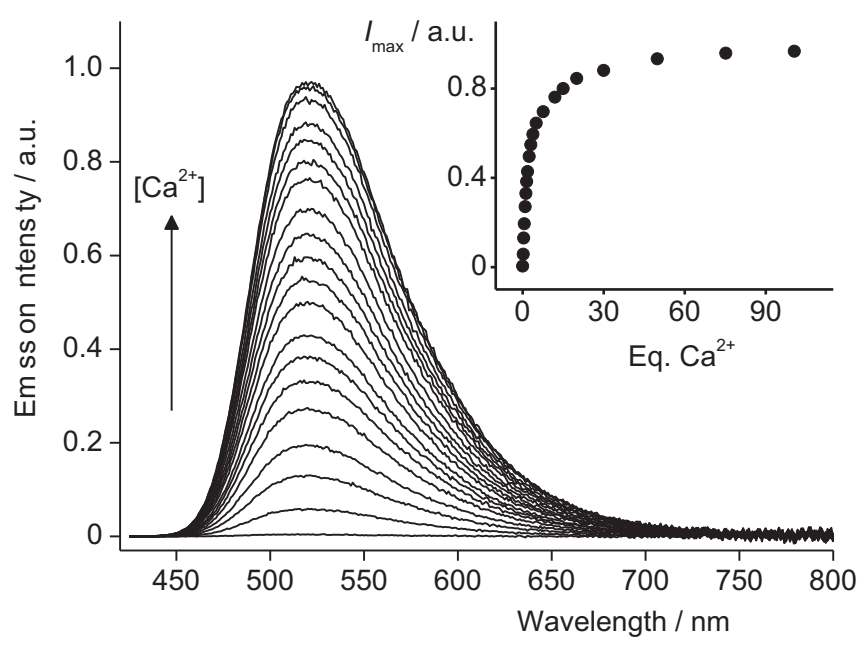

Fig. 4. The changes in the fluorescence spectrum of $\mathbf{1 b}$ in the presence of graduated amount of $\mathrm{Ca}\left(\mathrm{ClO}_{4}\right)_{2}$ in $\mathrm{MeCN}$ solution. $\mathrm{C}_{\mathrm{L}} \quad 5.010^{6} \mathrm{M}, \lambda_{\text {ex }} 420 \mathrm{~nm}$. The inserts show the fluorescence intensity at $\lambda_{\max }\left(I_{\max }\right)$ versus equivalents of $\mathrm{Ca}^{2+}$.

comparison with the ground state, enhances the nitrogen inversion rate responded for nonradiative deactivation pathway in these systems.

To predict the cation induced spectral effects in the case of crown containing derivatives $\mathbf{1 b}$ and $\mathbf{2} \mathbf{b}$ we studied the influence of the nature of the $N$ aryl group on photophysical properties. $N$ Phenyl substituted naphthalimides 1a and 2a were used for com parison. The introduction of electron donating dialkylamino group in the $N$ aryl fragment doesn't virtually change the position of absorption and emission maxima, whereas the fluorescence quantum yield drops sharply by more than two orders of magni tude (Table 1 ). The former observation is consistent with the $\mathrm{X}$ ray crystallography data of the compound $\mathbf{1 b}$ (Fig. 2) showing the dihedral angle between the naphthalimide moiety and $N$ aryl group to be close to $90^{\circ}$. Thus, one can conclude that the $\pi$ elec tronic interaction between these two fragments is very weak and negligible in the ground state.

The effect of decrease in emission intensity induced by the presence of azacrown ether groups in the compounds $\mathbf{1 b}$ and $\mathbf{2 b}$ can be explained in terms of PET quenching mechanism commonly used in the design of fluorophore-spacer-receptor signaling sys tems [2-5]. With this point of view, fast electron transfer process could be suggested between the electron rich $N$ aryl receptor in $\mathbf{1 b}$, 2b molecules and the excited naphthalimide fluorophore, which
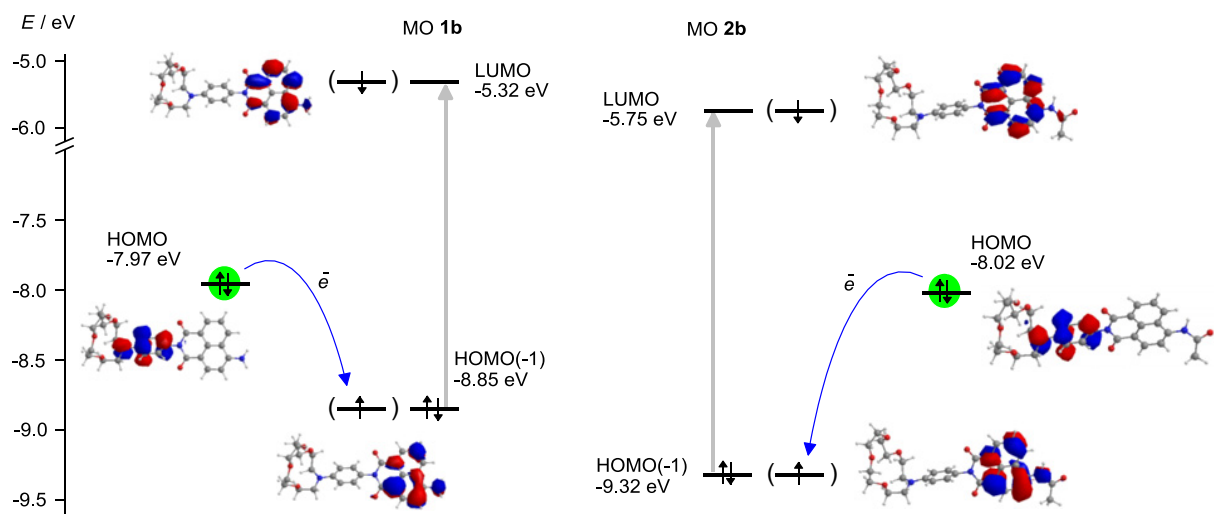

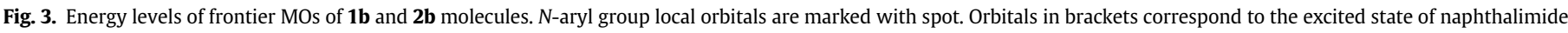
fluorophore. Vertical arrows describe lowest energy electronic transition (ICT transition) in naphthalimide fluorophore. 


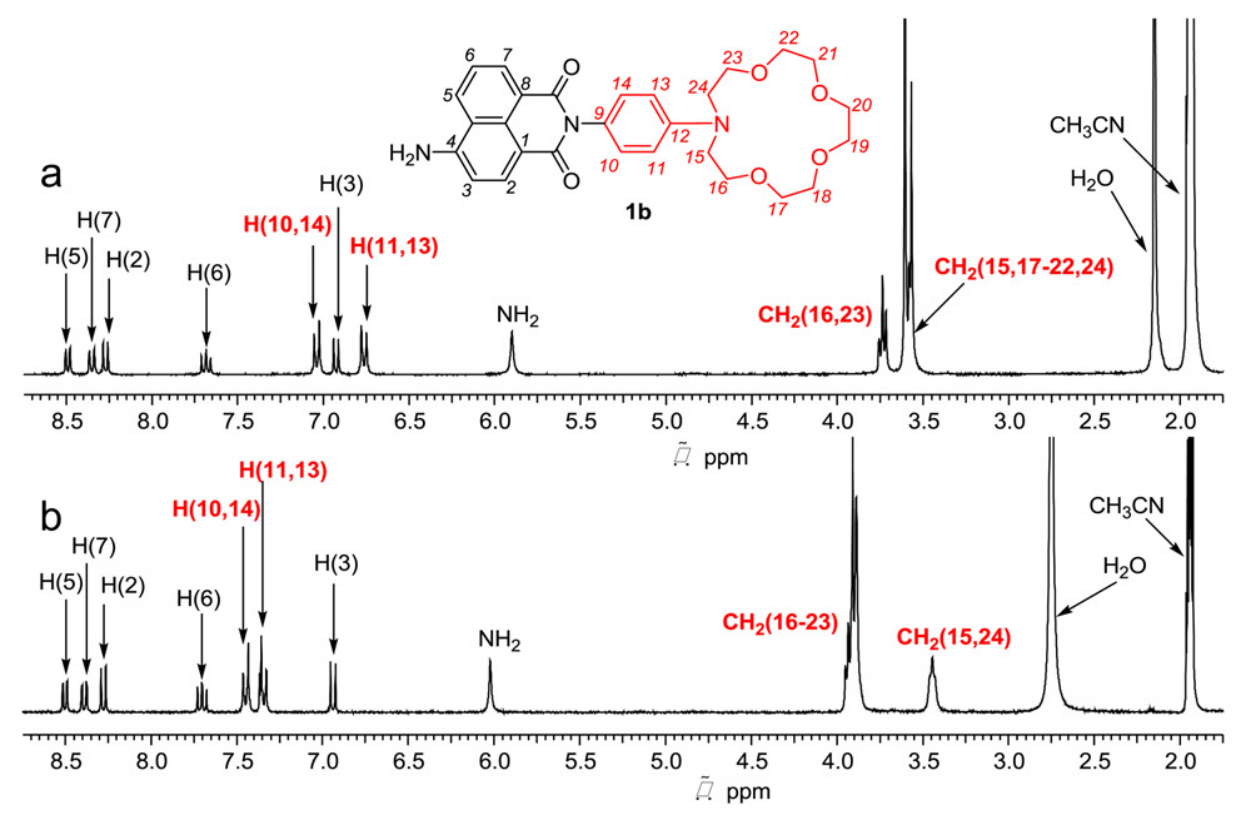

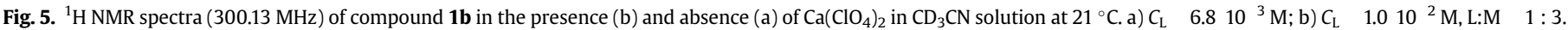

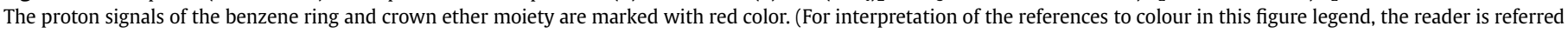
to the web version of this article.)

acts as an acceptor. Apparently, the $N$ aryl $\sigma$ bond is used as a spacer in this case. According to common principles in the con struction of fluorescent PET sensors [2], such spacer enables the required separation of donor and acceptor units due to their nearly orthogonal disposition in space and also links them together in a shortest way facilitating effective PET.

A striking evidence for existence of PET quenching pathway is also provided by the PM6 semiempirical calculations of the highest occupied and lowest unoccupied molecular orbitals (HOMO-LUMO) for the compounds $\mathbf{1 a}, \mathbf{2 a}$ and $\mathbf{1 b}, \mathbf{2 b}$. The calculation results are collected in Fig. 3 and Table 2. Fig. 3 shows the energy levels of the frontier orbitals of the naphthalimide fluorophore and local MOs of $N$ aryl groups (marked with spot) in the crown ether containing derivatives $\mathbf{1 b}, \mathbf{2} \mathbf{b}$. The assignment of local orbitals is based on their images, which are also depicted on Fig. 3 (see Fig. S2 in Supplementary data for orbital images of other molecules and complexes presented in Table 2). As it can be seen, both molecules exhibit the HOMO localized over the ben zene ring. The highest occupied MOs of naphthalimide fragments appear to be the $\operatorname{HOMO}(1)$ in whole molecules $\mathbf{1 b}, \mathbf{2 b}$ and therefore possess lower energy. Such situation corresponds to high driving force for PET process occurring between HOMO of $N$ aryl receptor and half occupied $\operatorname{HOMO}(1)$ in the excited state. In contrast, the energy level of the $N$ phenyl group HOMO in the compounds $\mathbf{1 a}, \mathbf{2 a}$ is lower relative to 4 amino or 4 amidonaphthalimide frontier orbitals (Table 2). Thus, in this case PET is hampered and a high fluorescence quantum yield value should be anticipated.

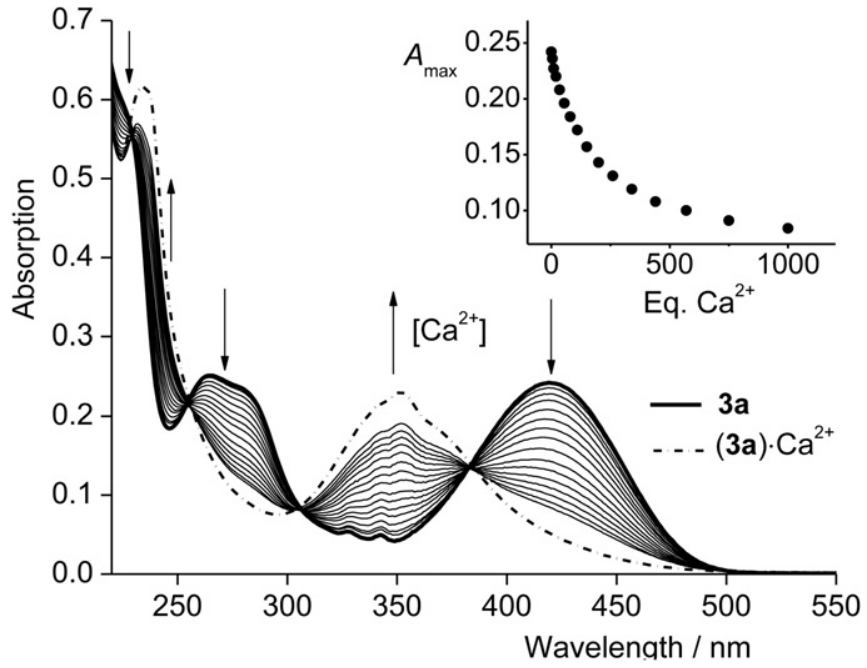

Fig. 6. The changes in absorption spectra of compound 3a in the presence of graduated amount of $\mathrm{Ca}\left(\mathrm{ClO}_{4}\right)_{2}$ in $\mathrm{MeCN}$. $\mathrm{C}_{\mathrm{L}} 2.010{ }^{5} \mathrm{M}$. The insert shows absorption at $420 \mathrm{~nm}$ $\left(A_{\max }\right)$ versus equivalents of $\mathrm{Ca}^{2+}$.

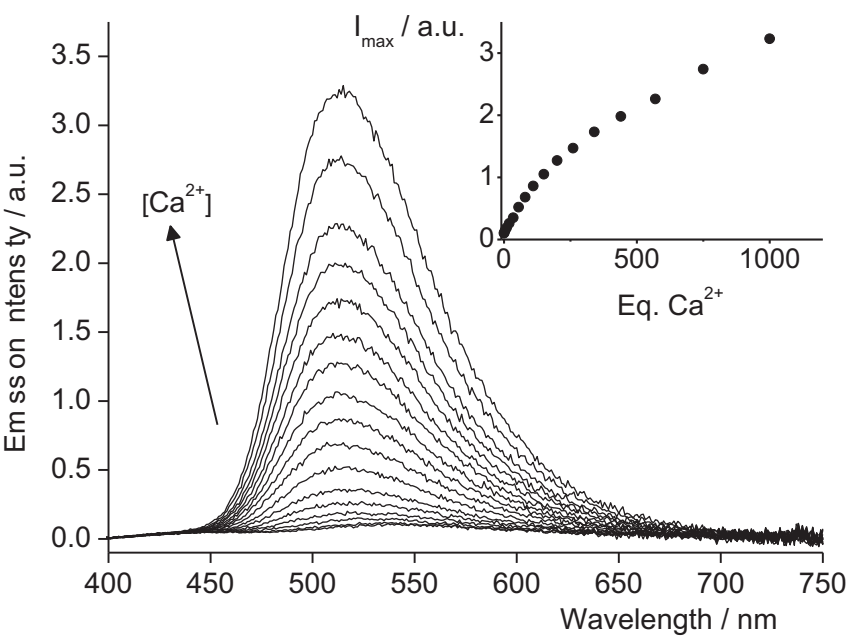

Fig. 7. The changes in emission spectra of compound $\mathbf{3 a}$ in the presence of graduated amount of $\mathrm{Ca}\left(\mathrm{ClO}_{4}\right)_{2}$ in $\mathrm{MeCN} . \mathrm{C}_{\mathrm{L}} 5.010^{6} \mathrm{M}, \lambda_{\mathrm{ex}} 355 \mathrm{~nm}$. The insert shows fluorescence intensity at $\lambda_{\max }\left(I_{\max }\right)$ versus equivalents of $\mathrm{Ca}^{2+}$. 


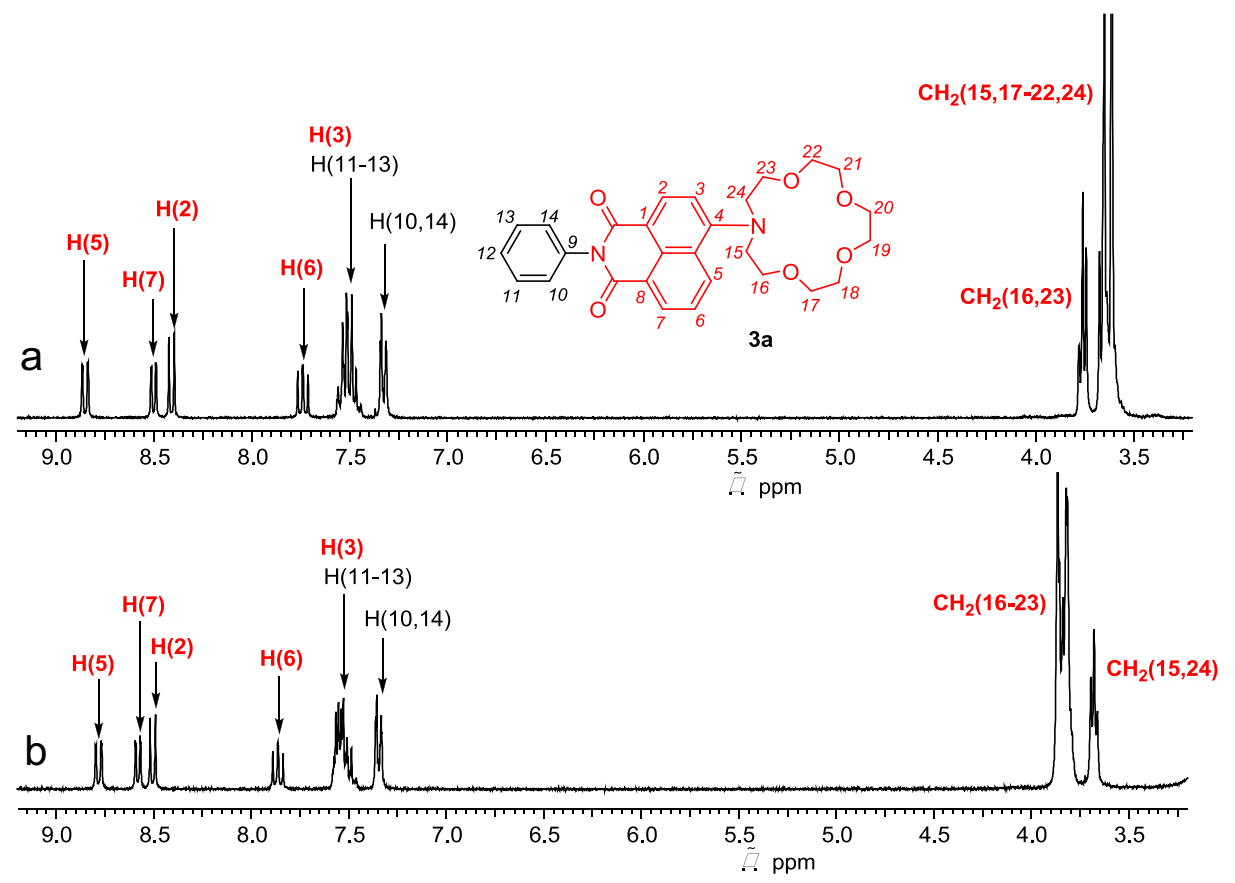

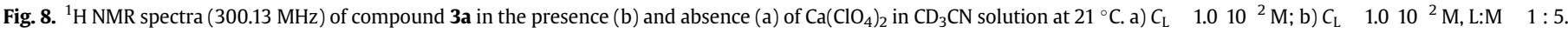

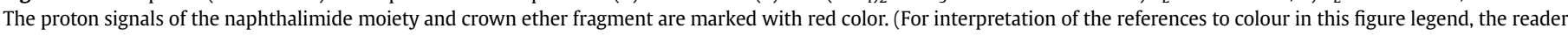
is referred to the web version of this article.)

\subsection{Complex formation studies of naphthalimides $1 b, 2 b$ and $3 a$}

Having established the spectral effects induced by the intro duction of aza 15 crown 5 fragment in $N$ phenyl 1,8 naphthalimides 1a, 2a, we examined the ability of crown ether derivatives $\mathbf{1 b}, \mathbf{2} \mathbf{b}$ and $\mathbf{3 a}$ to change their photophysical properties as a result of $\mathrm{Ca}^{2+}$ binding, the cation we used for the complexation studies. This cation was chosen because it has high affinity for aza 15 crown 5 ether in acetonitrile solution [51], which is convenient when studying cation induced optical effects.

Typically to fluorescent PET sensors, the addition of calcium perchlorate to the acetonitrile solutions of ligands $\mathbf{1 b}, \mathbf{2} \mathbf{b}$ does not lead to significant changes in the positions of absorption and fluorescence bands but results in pronounced increase in fluores cence intensity (Table 1). The fluorescence enhancement was assigned to coordination of $\mathrm{Ca}^{2+}$ with crown ether moiety in $\mathbf{1 b}, \mathbf{2 b}$ molecules. Such coordination should weaken the electron donor strength of $N$ phenylazacrown ether receptor thus decelerating the
PET quenching process. This is consistent with the fact that in complexes $(\mathbf{1 b}) \cdot \mathrm{Ca}^{2+}$ and $(\mathbf{2 b}) \cdot \mathrm{Ca}^{2+}$ the HOMOs of $N$ aryl groups possess lower energy relative to the free ligands (Table 2). Nearly identical position of $\lambda_{\max }^{\mathrm{abs}}$ and $\lambda_{\max }^{\mathrm{fl}}$ for $\mathbf{1 b}, \mathbf{2} \mathbf{b}$ in comparison with (1) $) \cdot \mathrm{Ca}^{2+},(\mathbf{2 b}) \cdot \mathrm{Ca}^{2+}$ is obviously due to efficient separation of $N$ arylazacrown ether receptor and naphthalimide chromophore by the $N-\operatorname{Ar} \sigma$ bond. The fluorescence emission spectra of compounds 1b and $\mathbf{2 b}$ were recorded in the presence of graduated amount of $\mathrm{Ca}\left(\mathrm{ClO}_{4}\right)_{2}$ in acetonitrile solution (see Fig. 4 for $\mathbf{1 b}$ and Fig. S3 in Supplementary data for $\mathbf{2 b}$ ) and the titration data were applied for the calculation of stability constants (Table 1 ).

The NMR analysis clearly confirmed the formation of complexes (Table 3). The addition of $\mathrm{Ca}^{2+}$ cations causes the downfield shift of the resonance proton signals of $N$ phenylazacrown ether part. The positions of the other proton signals stay unchanged. As an example, Fig. 5 shows cation induced changes in the ${ }^{1} \mathrm{H}$ NMR spectrum of $\mathbf{1 b}$. These results are in good agreement with the op tical data demonstrating only small changes in the position of

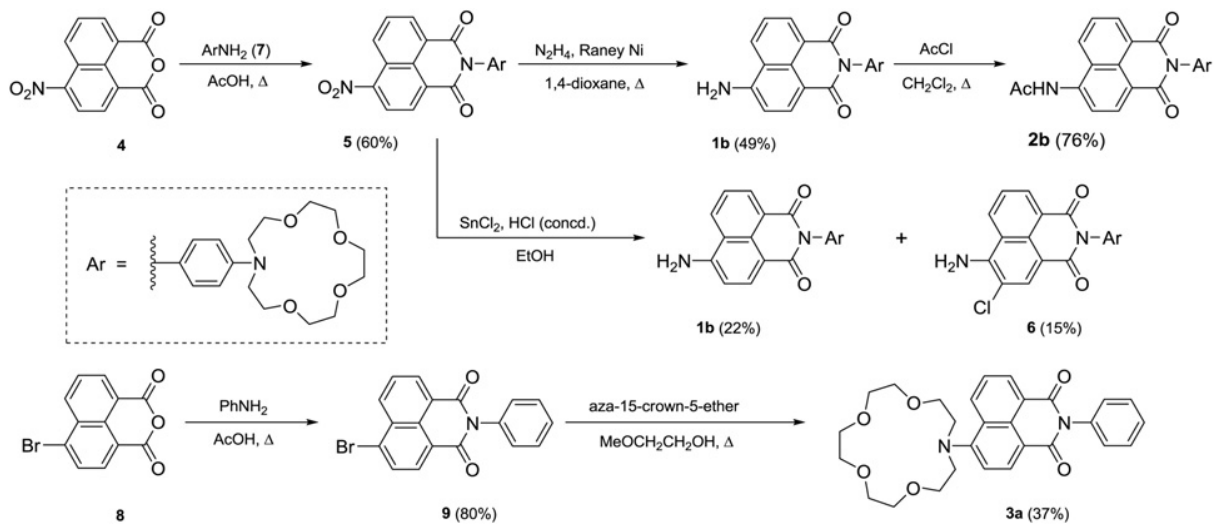

Scheme 2. Synthesis of the compounds $\mathbf{1 b}, \mathbf{2 b}$ and $\mathbf{3 a}$. 
Table 1

Optical properties and stability constants of $\mathbf{1 a}, \mathbf{1 b}, \mathbf{2} \mathbf{a}, \mathbf{2} \mathbf{b}, \mathbf{3} \mathbf{a}$ and their complexes in acetonitrile at $20^{\circ} \mathrm{C}$.

\begin{tabular}{llllll}
\hline & $\lambda_{\max }^{\text {abs }} / \mathrm{nm}$ & $\begin{array}{c}\varepsilon_{\lambda} \cdot 10^{3} / \mathrm{L} \\
\cdot \mathrm{mol}^{1} \cdot \cdot \mathrm{cm}^{1}\end{array}$ & $\lambda_{\max }^{\mathrm{fl}}\left(\lambda_{\mathrm{ex}}\right) / \mathrm{nm}$ & $\varphi^{\mathrm{fl}}$ & $\lg K^{\mathrm{a}}$ \\
\hline $\mathbf{1 a}$ & 417 & 12.9 & $518(420)$ & 0.55 & \\
$\mathbf{1 b}$ & 415 & 14.8 & $518(420)$ & 0.0024 & \\
$(\mathbf{1 b}) \cdot \mathrm{Ca}^{2+}$ & 420 & 14.4 & $520(420)$ & 0.43 & $4.94 \pm 0.02$ \\
$(\mathbf{1 b}) \cdot \mathrm{H}^{+}$ & 420 & 13.1 & $520(420)$ & 0.53 & $>7$ \\
$\mathbf{2 a}$ & 367 & 17.3 & $454(365)$ & 0.90 & \\
$\mathbf{2 b}$ & 366 & 15.9 & $454(365)$ & 0.0017 & \\
$(\mathbf{2 b}) \cdot \mathrm{Ca}^{2+}$ & 369 & 15.4 & $456(365)$ & 0.067 & $4.85 \pm 0.02$ \\
$(\mathbf{2 b}) \cdot \mathrm{H}^{+}$ & 369 & 15.3 & $457(365)$ & 0.89 & $>7$ \\
$\mathbf{3 a}$ & 420 & 12.1 & $540(420)$ & 0.027 & \\
$(\mathbf{3 a}) \cdot \mathrm{Ca}^{2+}$ & 352 & 11.5 & $513(340)$ & 0.26 & $2.38 \pm 0.03$ \\
\hline
\end{tabular}

a The dimension of $K$ is $\mathrm{M}^{1}$.

absorption and emission bands of ligands $\mathbf{1 b}$ and $\mathbf{2 b}$ upon the addition of $\mathrm{Ca}\left(\mathrm{ClO}_{4}\right)_{2}$.

As is evident from Table 1 , the fluorescence quantum yield value for complex $(\mathbf{1 b}) \cdot \mathrm{Ca}^{2+}$ is very close to that for $N$ phenyl naphthalimide 1a. In contrast, $(\mathbf{2 b}) \cdot \mathrm{Ca}^{2+}$ demonstrates considerable reduction of emission intensity as compared with $\mathbf{2 a}$. To explain this fact we proposed that in the case of $(\mathbf{2 b}) \cdot \mathrm{Ca}^{2+}$ PET interaction is only partially blocked due to the relatively low energy level of the highest occupied MO of 4 amidonaphthalimide fluorophore ( $9.50 \mathrm{eV}$, Table 2) in comparison with 4 aminonaphthalimide fragment $(9.04 \mathrm{eV})$ in $(\mathbf{1 b}) \cdot \mathrm{Ca}^{2+}$. Thus, the decrease in energy of the $N$ aryl localized $\mathrm{MO}$ in $\mathbf{1 b}$ occurring under complex formation with $\mathrm{Ca}^{2+}$ is high enough to ensure efficient quenching of PET interaction, whereas in $(\mathbf{2 b}) \cdot \mathrm{Ca}^{2+}$ PET inhibition is lower because the same changes in the donor ability of $N$ aryl group lead to more proximate disposition of HOMO and HOMO( 1) on energy scale (Table 2).

With the aim to confirm our assumption concerning partial inhibition of PET in the $(\mathbf{2 b}) \cdot \mathrm{Ca}^{2+}$, we measured photophysical characteristics of protonated forms of ligands $\mathbf{1 b}, \mathbf{2} \mathbf{b}$ (Table 1 ). The addition of $\mathrm{HClO}_{4}$ in acetonitrile solutions of $\mathbf{1 b}, \mathbf{2} \mathbf{b}$ results in for mation of highly fluorescent complexes $(\mathbf{1 b}) \cdot \mathrm{H}^{+}$and $(\mathbf{2 b}) \cdot \mathrm{H}^{+}$ similar in $\varphi^{\mathrm{fl}}$ values to 1a, 2a. In these complexes the lone electron pair of receptor's anilino nitrogen is fully engaged in coordination with the cation due to formation of $\mathrm{N}-\mathrm{H} \sigma$ bond, which breaks the conjugation, significantly lowers the potential energy of $N$ aryl group and thus completely quenches PET process in both cases. It should be also noted that in spite of the presence of two amino groups in the compound $\mathbf{1 b}$ only the anilino nitrogen of crown ether is subjected to protonation. This results from the observation that the position of $\lambda_{\max }^{\mathrm{abs}}$ and $\lambda_{\max }^{\mathrm{fl}}$ remains virtually unchanged upon addition of $\mathrm{HClO}_{4}$.

Unlike the typical PET sensors $\mathbf{1 b}$ and $\mathbf{2 b}$, the mechanism for binding signal transduction in compound $\mathbf{3 a}$ is supposed to be completely different. In this case the crown ether receptor's ni trogen is part of the $\pi$ system of naphthalimide signaling moiety. Thus, the complexation should give rise to dual response in both absorption and fluorescence emission spectra. Fig. 6 shows the influence of gradually increasing amounts of $\mathrm{Ca}^{2+}$ on the electronic
Table 3

The changes in ${ }^{1} \mathrm{H}$ NMR spectra of compounds $\mathbf{1 b}, \mathbf{2} \mathbf{b}$ induced by the presence of $\mathrm{Ca}\left(\mathrm{ClO}_{4}\right)_{2}$ in $\mathrm{CD}_{3} \mathrm{CN}$ solution.

\begin{tabular}{|c|c|c|c|c|c|}
\hline Hydrogen designation & $\mathrm{H}(10,14)$ & $\mathrm{H}(11,13)$ & $\mathrm{H}(15,24)$ & $\mathrm{H}(16,23)$ & $\mathrm{H}(17 \quad 22)$ \\
\hline$\Delta \delta / \mathrm{ppm}^{\mathrm{a}}$ Compound $\mathbf{1 b}$ & 0.41 & 0.57 & -0.14 & 0.19 & 0.31 \\
\hline Compound $\mathbf{2 b}$ & 0.39 & 0.57 & -0.13 & 0.20 & 0.32 \\
\hline
\end{tabular}

absorption spectra of $\mathbf{3 a}$ in acetonitrile solution. As it can be seen, the binding of metal ion causes an enhancement in the absorbance at $352 \mathrm{~nm}$ which corresponds to complex $(\mathbf{3 a}) \cdot \mathrm{Ca}^{2+}$ with decreasing the absorbance of the free ligand 3a centered at $420 \mathrm{~nm}$. Obviously, the blue shift of the long wavelength band is due to strong interaction of the crown ether nitrogen lone pair with the cation and reduced displacement of $\pi$ electron density from donor to acceptor unit in naphthalimide chromophore as a result.

The spectrophotometric titration data of 3a were applied for calculation of complex (3a) $\cdot \mathrm{Ca}^{2+}$ stability constant (Table 1 ) as well as its absorption spectra (marked with dash-dot line on Fig. 6). Considering the higher intensity at $352 \mathrm{~nm}$ of the calculated spectra as compared with that of the spectra recorded in the presence of 1000 equivalents of $\mathrm{Ca}^{2+}$, one could propose that ligand $3 \mathbf{a}$ is not fully engaged in a calcium complex even at such high excess of metal cation. Indeed, the constant stability value for $(\mathbf{3 a}) \cdot \mathrm{Ca}^{2+}$ appears to be lower by more than two orders of magnitude than in the case of $(\mathbf{1 b}) \cdot \mathrm{Ca}^{2+}$ and $(\mathbf{2 b}) \cdot \mathrm{Ca}^{2+}$, which is a consequence of conjugation between receptor's nitrogen with electron acceptor carbonyl groups of carboxyimide moiety.

The fluorescence spectra of the compound 3a exhibited pro nounced increase in emission intensity with the fluorescence band maxima blue shifted up to $27 \mathrm{~nm}$ in the presence of ascending amounts of $\mathrm{Ca}^{2+}$ (Fig. 7). Interestingly, the blue shift of the long wavelength absorption band was considerably higher $\left(\Delta \lambda_{\max }^{\mathrm{abs}} \lambda_{\max }^{\mathrm{abs}}\right.$ [3a] $\left.-\lambda_{\max }^{\text {abs }}\left[(\mathbf{3 a}) \cdot \mathrm{Ca}^{2+}\right] \quad 68 \mathrm{~nm}\right)$. This result indicates that, following the excitation, the nitrogen lone pair breaks its bond with the cation (as already reported for other fluoroionophores $[33,52,53])$, but the cation is not excluded from the macrocyclic cavity since it is still bonded with the oxygenated part of the crown ether. Such cation - nitrogen atom decoordination leads to ICT excited state in which the emission characteristics of naphthalimide chromophore fairly resemble that of the free ligand 3a. However, the proximity of positively charged metal ion reduces to some extent the electron donor properties of nitrogen atom due to elec trostatic interaction, thereby decreasing the push-pull character of ICT state. This explains the moderate hypsofluoric shift and enhancement of emission intensity. The data presented on Fig. 7 were also used for the calculation of complex stability constant and the obtained $\lg K$ value was essentially the same to the value derived from spectrophotometric titration experiment.

Upon addition of $\mathrm{Ca}\left(\mathrm{ClO}_{4}\right)_{2}$, the changes in ${ }^{1} \mathrm{H}$ NMR spectrum of compound $3 a$ in $\mathrm{CD}_{3} \mathrm{CN}$ were related only to downfield shift of naphthalimide and crown ether proton signals (Fig. 8), which in dicates the receptor's nitrogen lone pair is engaged in the cation coordination. Simultaneously, the position of $N$ phenyl group pro ton signals remains virtually unchanged.

Table 2

Energies (eV) of HOMO(-1), HOMO and LUMO of compounds $\mathbf{1}$ 2a,b and their complexes. ${ }^{\text {a }}$

\begin{tabular}{|c|c|c|c|c|c|c|c|c|}
\hline & $1 a$ & $1 b$ & $(\mathbf{1 b}) \cdot \mathrm{Ca}^{2+}$ & $(\mathbf{1 b}) \cdot \mathrm{H}^{+}$ & $2 a$ & $2 \mathbf{b}$ & $(\mathbf{2 b}) \cdot \mathrm{Ca}^{2+}$ & $(\mathbf{1 b}) \cdot \mathrm{H}^{+}$ \\
\hline LUMO & -5.34 & -5.32 & -5.84 & -5.51 & -6.05 & -5.75 & -6.17 & -5.82 \\
\hline HOMO & -8.85 & -7.97 & -9.04 & -9.00 & -9.34 & -8.02 & -9.50 & -9.46 \\
\hline $\operatorname{HOMO}(-1)$ & -9.86 & -8.85 & -9.73 & -10.01 & -9.96 & -9.32 & -9.74 & -10.14 \\
\hline
\end{tabular}

${ }^{\text {a }} \mathrm{N}$-Aryl group local orbitals are marked with gray color. 


\section{Conclusions}

In this work the synthesis and detailed investigation of optical characteristics and complexation ability of naphthalimide de rivatives bearing aza 15 crown 5 ether receptor in the $N$ aryl moiety and as a $\mathrm{C}(4)$ substituent of naphthalene ring were done. It was shown that the presence of crown ether group in the $\mathrm{N}$ aryl moiety of ligands $\mathbf{1 b}, \mathbf{2} \mathbf{b}$ leads to dramatic decrease of emission intensity with respect to $\mathrm{N}$ phenylnaphthalimides 1a, 2a due to efficient electron transfer between naphthalimide chromophore and receptor unit. Upon the addition of $\mathrm{Ca} 2+$ the lone pair of electrons of the crown ether receptor's nitrogen atom is engaged in cation binding; this suppresses PET process and causes fluores cence enhancement occurring at constant $\lambda_{\max }^{\text {abs }}$ and $\lambda_{\max }^{\mathrm{fl}}$ values. In the case of compound 3a receptor's nitrogen atom is a part of naphthalimide chromophore system, which is a structural feature of the ICT chemosensor. In contrast to PET sensors $\mathbf{1 b}, \mathbf{2} \mathbf{b}$, the co ordination of $\mathrm{Ca}^{2+}$ with 3a reduces ICT interaction and causes sig nificant hypsochromic and hypsofluoric shift. It was also shown that the various positions of the azacrown ether fragment in naphthalimide residue result not only in substantial difference in optical responses but also influence dramatically the affinity of the aza 15 crown 5 ether receptor for calcium cations. Noteworthy, the response of the compound $\mathbf{3 a}$ is useful both in absorption and emission spectroscopy. The strong wavelength shifts observed in the presence of $\mathrm{Ca}^{2+}$ make this system suitable for dual wavelength analysis in self calibrating measurements.

The presented results have shown that compounds $\mathbf{1 b}, \mathbf{2 b}$ and 3a can be of interest for the development of fluorescent and colorimetric chemosensors for different kinds of cationic analysis. Due to versatile structural modification on the crown ether moiety, selective probes based on aza 15 crown 5 containing 4 amino and 4 amidonaphthalimides for various metal cations can be pre pared. Related research is being carried out in our laboratory.

\section{Acknowledgments}

The work was supported by RFBR program $\mathcal{N} \circ 120300778$, program of Russian Ministry of Education and Science $\mathcal{N}$ 을 8533 and CNRS RAS exchange programs. Gediminas Jonusauskas thanks also the Région Aquitaine for financial support. Prof. Konstantin A. Lyssenko is gratefully acknowledged for $\mathrm{X}$ ray diffraction data.

\section{References}

[1] Winefordner JD. Trace analysis: spectroscopic methods for elements. New York: John Wiley \& Sons; 1976.

[2] Bissell RA, de Silva AP, Gunaratne HQN, Lynch PLM, Maguire GEM, McCoy CP, et al. Fluorescent PET (Photoinduced electron transfer) sensors. Top Curr Chem 1993;168:223 64

[3] Valeur B. Molecular fluorescence. principles and applications. Weinheim: Wiley-VCH; 2006.

[4] Callan J-F, de Silva AP, Magri DC. Luminescent sensors and switches in the early 21 st century. Tetrahedron 2005;61:8551 88 .

[5] Formica M, Fusi V, Giorgi L, Micheloni M. New fluorescent chemosensors for metal ions in solution. Coord Chem Rev 2012;256:170 92.

[6] Grabchev I, Konstantinova T. Synthesis of some polymerisable 1,8Naphthalimide derivatives for use as fluorescent brighteners. Dyes Pigm 1997;33:197 203.

[7] Grabchev I, Betcheva R. Copolymerization and photostabilization of methylmethacrylate with 1,8-naphthalimide fluorescent brighteners. J Photochem Photobiol A 2001;142:73 8 .

[8] Patrick LGF, Whiting A. Synthesis and application of some polycondensable fluorescent dyes. Dyes Pigm 2002;52:137 43.
[9] Martin E, Weigand R, Pardo A. Solvent dependence of the inhibition of intramolecular charge-transfer in $\mathrm{N}$-substituted 1,8-naphthalimide derivatives as dye lasers. J Luminesc 1996;68:157 64.

[10] Patrick LGF, Whiting A. Synthesis of some polymerisable fluorescent dyes. Dyes Pigm 2002;55:123 32

[11] May B, Poteau X, Yuan D, Brown RG. A study of a highly efficient resonance energy transfer between 7-N, N-diethylamino-4-methylcoumarin and 9-butyl-4-butylamino-1,8-naphthalimide. Dyes Pigm 1999;42:79 84.

12] Zhu W, Hu M, Yao R, Tian H. A novel family of twisted molecular luminescent materials containing carbazole unit for single-layer organic electroluminescent devices. J Photochem Photobiol A 2003;154:169 77.

[13] Tu G, Zhou Q, Cheng Y, Geng Y, Wang L, Ma D, et al. Synthesis and properties of polyfluorenes containing 1,8-naphthalimide moieties for white. Synth Met 2005; $152: 2336$

[14] Coya C, Blanco R, Juárez R, Gómez R, Martínez R, de Andrés A, et al. Synthesis and tunable emission of novel polyfluorene co-polymers with 1,8 naphthalimide pendant groups and application in a single layer single component white emitting device. Eur Polym J 2010;46:1778 89.

[15] Li Z, Yang Q Qian X. Novel thiazonaphthalimides as efficient antitumor and DNA photocleaving agents: effects of intercalation, side chains, and substituent groups. Bioorg Med Chem 2005;13:4864 70 .

[16] Grabchev I, Moneva I. Synthesis and properties of vinylic copolymers with fluorescent moieties as optical brighteners for liquid crystals. J Appl Polym Sci 1999;74:151 7

[17] Zhang Y, Zhu W, Wang W, Tian H, Su J, Wang W. Synthesis and nonlinear optical properties of rod-like luminescent materials containing Schiff-base and naphthalimide units. J Mater Chem 2002;12:1294 300.

[18] Stewart WW. Synthesis of 3,6-disulfonated 4-aminonaphthalimides. J Am Chem Soc 1981;103:7615 20.

[19] Sawa M, Hsu T-L, Itoh T, Sugiyama M, Hanson SR, Vogt PK, et al. Glycoproteomic probes for fluorescent imaging of fucosylated glycans in vivo. Proc Natl Acad Sci U S A 2006;103:12371 6

[20] Lin H-H, Chan Y-C, Chen J-W, Chang C-C. Aggregation-induced emission enhancement characteristics of naphthalimide derivatives and their applications in cell imaging. J Mater Chem 2011;21:3170 7.

[21] Li Z, Yang Q Chang R, Ma G, Chen M, Zhang W. N-Heteroaryl-1,8naphthalimide fluorescent sensor for water: molecular design, synthesis and properties. Dyes Pigm 2011;88:307 14

[22] Magalhães JL, Pereira RV, Triboni ER, Filho PB, Gehlen MH, Nart FC. Solven effect on the photophysical properties of 4-phenoxy-N-methyl-1,8naphthalimide. J Photochem Photobiol A 2006;183:165 70.

[23] Gan J, Chen K, Chang C-P, Tian H. Luminescent properties and photo-induced electron transfer of naphthalimides with piperazine substituent. Dyes Pigm 2003;57:21 8.

[24] Li Z-Z, Niu C-G, Zeng G-M, Liu Y-G, Gao P-F, Huang G-H, et al. A novel fluorescence ratiometric $\mathrm{pH}$ sensor based on covalently immobilized piperazinyl-1,8-napthalimide and benzothioxanthene. Sens Actuators B 2006;114: 30815.

[25] Wang J, Xiao Y, Zhang Z, Qian X, Yang Y, Xu Q. A pH-resistant Zn(II) sensor derived from 4-aminonaphthalimide: design, synthesis and intracellular applications. J Mater Chem 2005;15:2836 9.

[26] Cui D, Qian X, Liu F, Zhang R. Novel fluorescent pH sensors based on intramolecular hydrogen bonding ability of naphthalimide. Org Lett 2004;6: 275760.

[27] Xu Z, Yoon J, Spring DR. A selective and ratiometric $\mathrm{Cu}^{2+}$ fluorescent probe based on naphthalimide excimer monomer switching. Chem Commun 2010;46:2563 5 .

28] He H, Mortellaro MA, Leiner MJP, Young ST, Fraatz RJ, Tusa JK. A fluorescent chemosensor for sodium based on photoinduced electron transfer. Anal Chem 2003;75:549 55.

[29] Nandhikonda P, Begaye MP, Heagy MD. Highly water-soluble, OFF ON, dual fluorescent probes for sodium and potassium ions. Tetrahedron Lett 2009;50: 245961.

[30] Chen T, Zhu W, Xu Y, Zhang S, Zhang X, Qian X. A thioether-rich crown-based highly selective fluorescent sensor for $\mathrm{Hg}^{2+}$ and $\mathrm{Ag}^{+}$in aqueous solution. Dalton Trans 2010;39:1316 20.

[31] Bricks JL, Kovalchuk A, Trieflinger C, Nofz M, Büschel M, Tolmachev AI, et al. On the development of sensor molecules that display $\mathrm{Fe}^{\mathrm{III}}$-amplified fluorescence. J Am Chem Soc 2005;127:13522 9.

[32] Rurack K, Resch-Genger U, Bricks JL, Spieles M. Cation-triggered 'switching on' of the red/near infra-red (NIR) fluorescence of rigid fluorophore spacer receptor ionophores. Chem Commun 2000:2103 4.

[33] Cosnard F, Wintgens V. A new fluoroionophore derived from 4-amino-NMethyl-1,8-Naphthalimide. Tetrahedron Lett 1998;39:2751 4

[34] Panchenko PA, Fedorov YV, Perevalov VP, Jonusauskas G, Fedorova OA Cation-dependent fluorescent properties of naphthalimide derivatives with N-benzocrown ether fragment. J Phys Chem A 2010;114:4118 22.

[35] Okazaki M, Tanaka T, Taniguchi S. Synthesis of 5-nitroacenaphthene. Chem Abstr 1957;51:8050. [J. Soc. Organ. Synth. Chem., Jpn. 1956;14(5): $3446]$

[36] Okazaki M, Suhara Y, Fujiyama M. Synthesis of 4-dinitronaphthalic acid. Chem Abstr 1957;51:8050. [J. Soc. Organ. Synth. Chem., Jpn. 1956;14(6):394 8].

[37] Nakaya K, Funabiki K, Muramatsu H, Shibata K, Matsui M. N-Aryl-1,8 naphthalimides as highly sensitive fluorescent labeling reagents for carnitine. Dyes Pigm 1999;43:2359. 
[38] Sergeeva AN, Panchenko PA, Fedorov YV, Fedorova OA. Synthesis and sensor propeties of crown-containing derivatives of 4-(1,5-diphenyl- $\Delta^{2}$ Pyrazolin-3-yl)-1,8-naphthalimide. Protec Met Phys Chem Surf 2012;48(5): 52433.

[39] Perry DL. Handbook of inorganic compounds. Boca Raton: CRC Press; 2011.

[40] Vogel AI. Furniss BS, Hannaford AJ, Smith PWG, Tatchell AR, editors. Vogel's textbook of practical organic chemistry. New York: John Wiley \& Sons; 1989.

[41] Nad S, Kumbhakar M, Pal H. Photophysical properties of coumarin-152 and Coumarin-481 dyes: unusual behavior in nonpolar and in higher polarity solvents. J Phys Chem A 2003;107:4808 16.

[42] Renschler CL, Harrah LA. Determination of quantum yields of fluorescence by optimizing the fluorescence intensity. Anal Chem 1983;55:798 800 .

[43] Connors KA. Binding constants: the measurement of molecular complex stability. New York: John Wiley \& Sons; 1987.

[44] Beck MT, Nagypál I. Chemistry of complex equilibria. New York: John Wiley \& Sons; 1990

45] Sheldrick GM. A short history of SHELX. Acta Cryst A 2008;64:112 22.

[46] Stewart JJP. Optimization of parameters for semiempirical methods $V$ : modification of NDDO approximations and application to 70 elements. J Mol Model 2007;13:1173 213.
[47] Panchenko PA, Fedorov YV, Fedorova OA, Perevalov VP, Jonusauskas G. Synthesis and spectral properties of 4-amino- and 4-acetylamino-N-arylnaphthalimides containing electron-donating groups in the $\mathrm{N}$-aryl substituent. Russ Chem Bull 2009;58(6):1233 40.

[48] Krasovitskii BM, Bolotin BM. Organic luminescent materials. New York: Wiley-VCH; 1988.

[49] Alexiou MS, Tychopoulos V, Ghorbnian S, Tyman JHP, Brown RG, Brittain PI. The UV Visible absorption and fluorescence of some substituted 1,8-Naphthalimide and naphthalic anhydrides. J Chem Soc Perkin Trans 1990;2:837 42.

[50] Saha S, Samanta A. Influence of the structure of the amino group and polarity of the medium on the photophysical behavior of 4-Amino-1,8-naphthalimide derivatives. J Phys Chem A 2002;106:4763 71

[51] Fedorova OA, Strokach YP, Gromov SP, Koshkin AV, Valova TM, Alfimov MV, et al. Effect of metal cations on the photochromic properties of spironaphthoxazines conjugated with aza-15(18)-crown-5(6) ethers. New J Chem 2002;26:1137 45.

[52] Mathevet R, Jonusauskas G, Rullière C, Létard J-F, Lapouyade R. Picosecond transient absorption as monitor of the stepwise cation-macrocycle decoordination in the excited Singlet state of 4-(N-monoaza-15-crow-5)-4'-cyanostilbene. J Phys Chem 1995;99:15709 13

[53] Létard J-F, Lapouyade R, Rettig W. Synthesis and photophysical study of 4-(Nmonoaza-15-crown-5) stilbenes forming TICT states and their complexation with cations. Pure Appl Chem 1993;65:1705 12 